\title{
The auditory organization of speech and other sources in listeners and computational models
}

\author{
Martin Cooke ${ }^{\mathrm{a}, *}$, Daniel P.W. Ellis ${ }^{\mathrm{b}}$ \\ ${ }^{a}$ Department of Computer Science, University of Sheffield, UK \\ ${ }^{\mathrm{b}}$ Department of Electrical Engineering, Columbia University, New York, USA
}

Received 25 March 2000; received in revised form 5 June 2000; accepted 14 June 2000

\begin{abstract}
Speech is typically perceived against a background of other sounds. Listeners are adept at extracting target sources from the acoustic mixture reaching the ears. The auditory scene analysis (ASA) account holds that this feat is the result of a two-stage process. In the first-stage, sound is decomposed into collections of fragments in several dimensions. Subsequent processes of perceptual organization reassemble these fragments, based on cues indicating common source of origin which are interpreted in the light of prior experience. In this way, the decomposed auditory scene is processed to extract coherent evidence for one or more sources. Auditory scene analysis in listeners has been studied for several decades and recent years have seen a steady accumulation of computational models of perceptual organization. The purpose of this review is to describe the evidence for the nature of auditory organization in listeners and to explore the computational models which have been motivated by such evidence. The primary focus is on speech rather than on sources such as polyphonic music or non-speech ambient backgrounds, although all these domains are equally amenable to auditory organization. The review includes a discussion of the relationship between auditory scene analysis and alternative approaches to sound source segregation. (c) 2001 Elsevier Science B.V. All rights reserved.
\end{abstract}

Keywords: Auditory scene analysis; Speech perception; Streaming; Auditory induction; Double vowels; Robust ASR

\section{Introduction}

Speech is typically perceived against a background of other sounds. The acoustic mixture reaching the ears is processed to enable constituent sounds to be heard and recognized as distinct entities. While the auditory system may not always succeed in this goal, the range of situations in which spoken communication is possible in the

\footnotetext{
* Corresponding author. Tel.: +44-114-222-1822; fax: +44114-222-1810.

E-mail addresses: m.cooke@dcs.shef.ac.uk (M. Cooke), dpwe@icsi.berkeley.edu (D.P.W. Ellis).
}

presence of competing sources highlights the flexibility and robustness of human speech perception. The background against which a conversation is carried out is made up of acoustic intrusions which overlap in both time and frequency with the target speech. The background may consist of other utterances whose fundamental frequency and formant contours occupy similar regions to those of the target. Target and background may contain similar kinds of envelope modulations, and can arrive from similar locations in space. Sometimes, the background will be characterized by high-intensity onsets which completely overwhelm the target conversation. Fig. 1 depicts auditory 

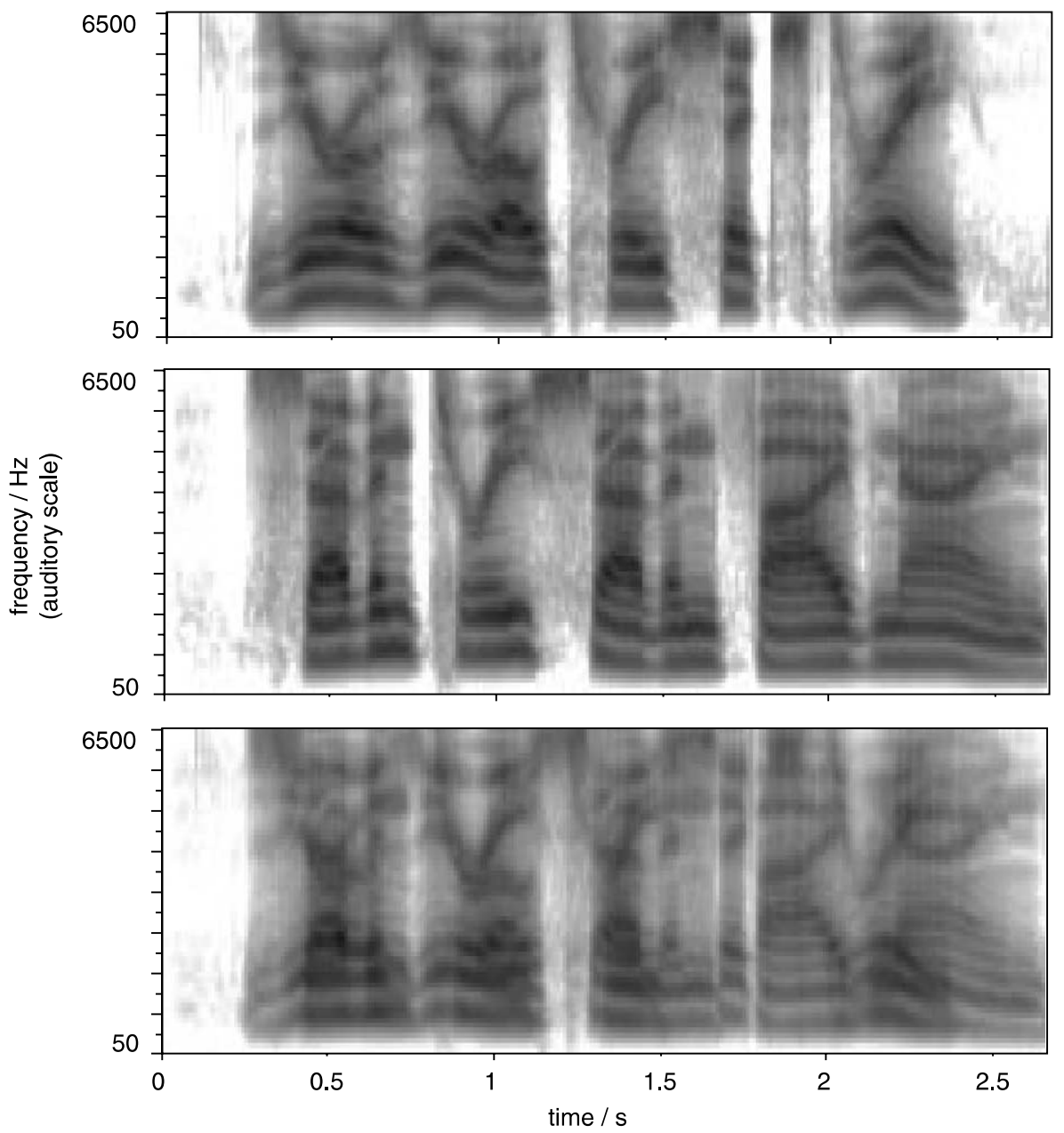

Fig. 1. Auditory spectrograms of spoken digit sequences. Upper: "zero zero three six three". Middle: "seven three seven five nine". Lower: mixed signal. Gray-levels are proportional to log-energies at the output of a bank of 64 gammatone filters, equally spaced on an auditory scale (ERB-rate) from 50 to $6500 \mathrm{~Hz}$.

spectrograms for a mixture of two digit sequences whose constituents differ in onset time, fundamental frequency contour and formant structure but which are still sufficiently similar in these properties to make visual separation difficult.

\subsection{Terminology}

Bregman (1984, 1990) draws a distinction between an acoustic source - a single physical system giving rise to a particular pattern of sound waves and an auditory stream which denotes the abstract, conceptual effect it has in the mind of the listener.
Listeners have to solve an auditory scene analysis (ASA) problem in order to extract one or more relevant auditory streams from the mixture of sources which contribute to their acoustic environment.

On entering the ear, the signal undergoes several transformations, leaving the periphery as patterns of nerve-firings which may be considered as representations of all or part of the sound. Features of these representations which are used to achieve a particular end are called cues. Different theories for the organization of sound have varying assumptions of which features are actually employed as cues. 
Sound sources may differ in all kinds of properties such as location, instantaneous fundamental frequency, or the patterns of energy envelope modulation in different frequency bands. If it is possible to extract these potential cues with sufficient reliability and sufficiently often, the auditory system can group those parts of the mixture possessing similar values of each property. This affords listeners a basis for organizing into a coherent whole the sound fragments which have a common origin. This style of processing is often described as bottom-up or primitive.

In addition to primitive grouping processes, listeners can exploit prior familiarity with the patterns of spoken language or other sources. For speech, these regularities manifest themselves at a number of levels, from the sub-syllabic to the sentential. Speech represents a rich and redundant encoding of information, so prior experience can help to fill in those parts of the signal that are masked or otherwise distorted. Such top-down processes have been termed schema-driven mechanisms (Bregman, 1990).

Early auditory signal processing involves at least two forms of decomposition. First, the signal is subject to a spectral decomposition into separate frequency bands by the cochlea - an organizational axis maintained throughout many later processing stages. Second, it appears that different properties are extracted in distinct auditory maps (Moore, 1987), or distributions of specific signal features over an array of neural elements. Consequently, information arising from a single acoustic source is distributed both across cochleotopic frequency and between several auditory brain centers. For instance, voiced speech gives rise to a series of harmonically related spectral peaks in the relatively narrow-band cochlear filters at low frequencies. The upper spectrum might contain envelope modulations at the voicing fundamental frequency $\left(f_{0}\right)$ as reflected in the temporal envelope, or equivalently as caused by the interaction of neighboring harmonics in the response area of each broader auditory filter. This periodicity will also appear in the fine time structure of the lower bands. Moore (1997, Fig. 5.6), depicts some of these properties of the auditory filter bank response to periodic sounds. It is possible that de- tection and processing of harmonic peaks, envelope, and fine structure are carried out in distinct auditory maps.

This twofold separation (by frequency channel and cue class) has practical appeal: since different sources in an acoustic mixture may dominate distinct spectral regions, spectral decomposition is an elementary first step in signal separation. Functional decomposition into distinct auditory maps allows the deployment of special-purpose processing hardware to extract different signal properties such as $f_{0}$ and location, including the possibility of using several complementary approaches for each of these properties.

In the light of this fragmentation of the original sound into several features defined over multiple dimensions, it is inadequate to make statements such as "sound components with a common fundamental are grouped together". We must also address the specifics of grouping, such as how the components are defined, and which of the several alternative mechanisms are used to extract and recognize their common fundamental, for instance. There is the interplay between primitive and schema-driven grouping to be examined, and it is necessary to contrast grouping of local features within auditory maps with grouping of features corresponding to the same source represented in different maps.

\subsection{Summary of grouping cues}

Table 1 summarizes the many experimental investigations of grouping. The organization of the table reflects the idea that each property of an acoustic source produces a number of auditory consequences, each of which represents a potential grouping cue. Darwin and Carlyon (1995) provide a quantitative tabulation of some of these investigations and demonstrate that grouping, rather than being "all-or-nothing", occurs at different feature magnitudes depending on the measure used.

The availability of numerous cues for sound organization accommodates situations in which any one of them may fail to indicate the correct grouping, but also creates a problem for higher auditory levels due to the possibility of inconsis- 


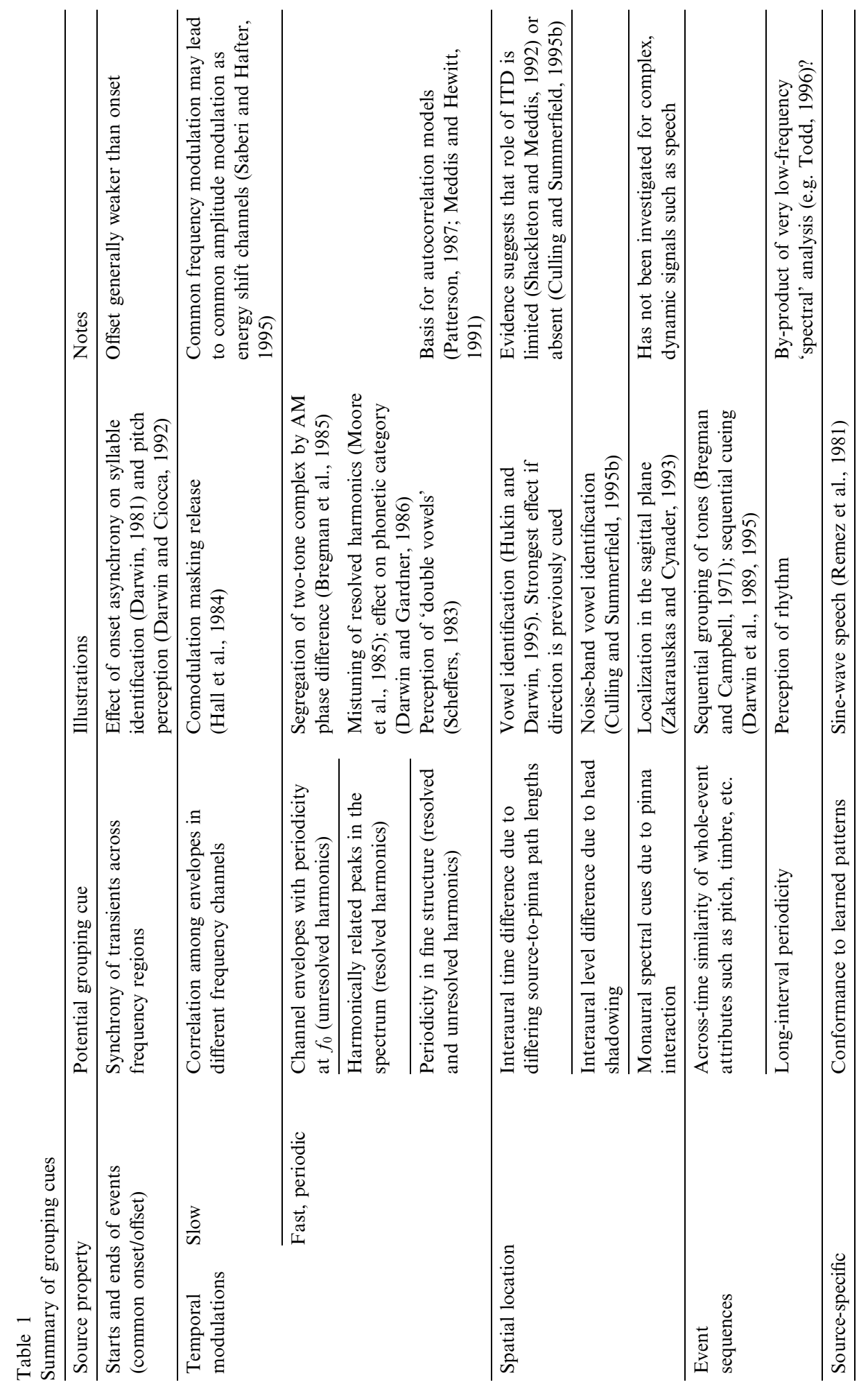


tent or conflicting cues. Investigations of conflicts between cues such as frequency proximity and ear of presentation (Deutsch, 1975) or onset asynchrony and mistuning (Darwin and Ciocca, 1992; Ciocca and Darwin, 1993) can provide valuable insight into high-level audition; we will return to this in Section 5.

Some signal features that have been proposed as potential grouping cues do not appear in Table 1. Foremost among these is the common frequency modulation imposed on the harmonics in voiced speech. There is little evidence for an independent effect of grouping by common FM over and above that provided by instantaneous harmonicity (Gardner and Darwin, 1986; Summerfield and Culling, 1992; Carlyon, 1994), although the presence of FM can make vowels more prominent against a background of unmodulated sounds (McAdams, 1984).

\subsection{Review organization}

Section 2 provides a chronological review of important developments in auditory organization. Sections 3-6 reflect a systematic progression from lower to higher levels of stimulus complexity. Section 3 deals with simple tonal configurations, while Section 4 examines the extensive experimental and modeling work employing simultaneous synthetic vowels. Sections 5 and 6 explore the role of bottom-up and top-down factors in processing natural utterances. Within each section, relevant perceptual evidence for organization in listeners is considered, followed by details of algorithms which attempt to replicate the effects in machines. The review concludes with a discussion of the major issues facing CASA and its relation to other approaches to source segregation.

\section{Auditory organization: development of the field}

\subsection{Listeners}

Cherry (1953) provides one of the earliest accounts of the problem faced by listeners when presented with simultaneous utterances. Speculating on what he termed the "cocktail party prob- lem", he considered possible cues to its solution location, lip-reading, mean pitch differences, different speeds, male/female speaking voice, accents and the like. Cherry demonstrated the relative ease with which one of a pair of simultaneous sentences could be repeated when the messages were sent to different ears. In a refinement of this strategy, Broadbent and Ladefoged (1957) have employed synthetic, two-formant speech to examine the roles of both ear of presentation and fundamental frequency on perceptual fusion, as reflected by the number of voices heard by listeners. They found that fusion occurred even when the two formants were sent to different ears, but that giving the two formants sufficiently different fundamental frequencies prevented fusion. Their findings not only demonstrated a clear role for fundamental frequency differences in perceptual organization, but were an early anticipation of the interactions that occur when multiple cues for grouping are placed in opposition, a recurrent theme in studies of grouping and segregation. Broadbent and Ladefoged were among the first authors to recognize the computational problem posed by hearing, noting that perception in the presence of other sounds represents the normal, everyday mode for spoken language processing.

A different approach to the study of everyday speech perception came with the finding by Warren (1970) that listeners were unaware of the absence of short segments of sentences which had been replaced by a louder noise. This phenomenon was termed the phonemic restoration effect. Later work (Warren et al., 1972) generalized its application to non-speech signals. Phonemic restoration is now considered as a special instance of a collection of auditory induction effects, including induction between ears and across frequencies. Section 6 discusses such induction effects.

Warren's work was an important demonstration that the auditory system is not simply a passive conduit for sensory information, but is engaged in active interpretation, and could in consequence generate illusions or otherwise impose structure beyond the manifest signal. Bregman and Campbell (1971) have studied the dichotomy, long exploited in music, between hearing a sequence of alternating high and low 
tones as a single stream or as two streams, each composed of all the tones of one pitch. They showed that the interpretation depended consistently on factors such as frequency difference and repetition rate. Section 3 describes some of these "streaming" experiments.

Much of this early work on streaming employed simple tonal stimuli, although some studies used speech-like sounds and demonstrated similar effects of factors such as spectral dissimilarity on streaming (Cole and Scott, 1973), and pitch and formant continuity on speech coherence (Darwin and Bethell-Fox, 1977). These studies used repeated sequences to induce segregation, raising the question of whether grouping cues uncovered in such experiments are relevant to everyday speech perception. Darwin's (1981) attempt to find evidence for grouping in speech was a turning point. His experiments were based on Cutting's (1976) demonstration that listeners could correctly identify syllables when the formants were presented to different ears (e.g. the lowest formant, F1, to the left ear, with the right ear receiving F2 and F3) even when different fundamental frequencies were used for each ear. Darwin systematically varied $f_{0}$ and onset time between the two ears, finding only one stimulus for which these manipulations affected the phonetic categories perceived by listeners. This synthetic four-formant complex had the unusual property of resembling two equally plausible syllables: it was heard as "ru" if all formants were integrated, but as "li" if F2 was excluded into a separately perceived source. By testing which syllable was heard for a particular condition, Darwin was able to study how variations in $f_{0}$ and onset time determined the integration or exclusion of the F2 signal.

The demonstrations by Cutting (1976) and Darwin (1981) that phonetic interpretations could often override conflicting cues for perceptual organization led to the realization that explorations of grouping need to be performed in a phonetically neutral context. Over the next few years, a series of refinements and new paradigms enabled a much closer analysis of the role of perceptual grouping in speech, with the spotlight on the identification of synthetic stationary vowels. Darwin (1984) exploited the fact that moving the center of the F1 resonance of [I] from 375 to $500 \mathrm{~Hz}$ shifts its perceptual category to [e] (e.g. "bit" becomes "bet"). Manipulating the properties of the individual harmonics that define F1 and measuring the perceived category (and hence the perceived F1 center) gave a very sensitive measure of the extent to which the modified harmonic was integrated with the rest of the complex. These experiments demonstrated that onset or offset asynchrony could reduce the contribution that a harmonic makes to vowel quality. Darwin and Gardner (1986) again used the $[\mathrm{I}]-[\mathrm{e}]$ continuum, this time showing that a mistuned harmonic contributes less to vowel quality, resembling the way in which it can be excluded from the computation of pitch (Moore et al., 1985).

An alternative approach to the study of grouping in speech was introduced by Scheffers (1983). He asked listeners to identify both constituents in pairs of simultaneous synthetic vowels. This double-vowel task, as it came to be known, has proved to be a fertile paradigm for the study of auditory perceptual organization and is reviewed in Section 4.

By 1990, a significant body of perceptual studies of auditory fusion and segregation had accumulated, consolidated by Bregman's (1990) comprehensive monograph. Many properties of sound sources considered as potential features for organization have been investigated, including findings of the failure of grouping under circumstances which might otherwise have been thought to promote it. For example, changes in $f_{0}$ lead to correlated changes in harmonic frequencies, known as common frequency modulation (FM). Gardner et al. (1989), using the "ru"-"li" paradigm, found no effect of incoherent FM in segregating F2 from the remainder of the syllable.

More recently, researchers have investigated the relationship of grouping to other aspects of auditory function, such as the determination of pitch, location, or phonetic quality of a sound source. Darwin and Carlyon (1995) document the taskdependent nature of the cue manipulation required to reveal grouping effects. For example, in the tasks of detection, identification as a separate source, determination of pitch, vowel classification, speech separation, and lateralization, the 
relevant degree of mistuning for a single harmonic varies from $1 \%$ to $10 \%$. Similarly, the amount of onset or offset asynchrony required over a range of tasks can vary from a few milliseconds for detection to several hundreds of milliseconds for tasks involving pitch and vowel identification.

\subsection{Models}

One of the earliest computational attempts at speech separation was the signal-processing approach of Parsons (1976). Although Parsons was not motivated by auditory findings, his system served to define - and partially solve - some of the issues which have since become central for computational auditory scene analysis (CASA) systems operating on voiced speech. These problems include the determination of multiple pitches, the handling of harmonics from different sources that fall close to one another, and the tracking of fundamental frequency contours which may cross. Parsons described the separation of voiced speech as the principal sub-problem, and his system set about solving it by identifying two sets of harmonic peaks in a standard fixed-bandwidth Fourier-transform spectrum, estimating their underlying fundamental frequencies and tracking their evolution through time.

Lyon (1983) - influenced by Jeffress' (1948) proposal for an interaural delay line mechanism presented a computational model of binaural localization and separation which performed a cross-correlation of the outputs of cochlear simulations for opposing ears. Lyon used the term "correlagram" to describe the cross-correlation representation (the term "correlogram" has since come to refer primarily to an autocorrelation analysis) and demonstrated separation of a short speech signal from an impulsive sound generated by striking a ping-pong ball. Weintraub (1985) was the first to design a system with an explicit auditory motivation to tackle the more difficult problem of sentence separation. His pitch-based separation system was inspired by Licklider's (1951) postulation of neural periodicity sensors built from delays and coincidence detectors.

These early demonstrations illustrated the engineering potential of cues such as pitch and in- teraural differences, but they lacked quantitative evaluation. One of the first studies to do so was the evaluation by Stubbs and Summerfield (1988) of two algorithms for the separation of voices based on a difference in fundamental frequency in a single channel. One approach operated by attenuating the pitch peak corresponding to the interfering voice through filtering the cepstrum of the mixed signal. The other was similar to Parsons' (1976) harmonic selection scheme. Stubbs and Summerfield used synthetic vowel pairs in one task and real $\mathrm{CV}$ words masked by synthetic vowels in another, and resynthesized signals in which the target speech sounds had been enhanced by each algorithm. They evaluated the extent to which the enhanced speech was more intelligible to normal listeners as well as those with hearing impairments.

The decade since Weintraub's system has witnessed a proliferation of modeling attempts, many of which are described in the following sections.

\section{The streaming effect}

\subsection{Listeners}

A sequence of alternating high- and low-frequency tones can result in the perception of either one or two coherent patterns or streams (Miller and Heise, 1950; Bregman and Campbell, 1971). Factors influencing segregation into streams are discussed at length in Bregman (1990, Chapter 2) and summarized below:

- Frequency separation: If the frequency difference between alternating high and low tones is progressively increased, the perception of a continuously alternating pitch (the "trill") changes to that of two separate tone streams. The frequency separation at which this occurs was termed the "trill threshold" by Miller and Heise (1950). Using a different measure of streaming based on rhythm, van Noorden (1975) demonstrated that the streaming effect could better be described by two thresholds, a lower one (the "fission boundary") below which the tones always formed a single stream, and a larger one (the "temporal coherence boundary") beyond which the tones always separated into two 
streams. In the intervening range of frequency separations, listeners could alternate between hearing one or two streams.

- Rate of alternation: van Noorden (1975) mapped out the fission and temporal coherence boundaries as a function of tone onset-to-onset interval. At short tone repetition times $(60 \mathrm{~ms})$, the boundaries are quite close, while for larger intervals $(150 \mathrm{~ms})$, the boundaries are far apart. However, the fission boundary remains low and is largely unaffected by tone repetition time, suggesting that while it is relatively easy to try to hear two streams, it is very difficult to hold on to a single stream at high repetition speeds.

- Duration: Sequences are heard as a single stream until sufficient evidence is gathered to split them. Thus, Bregman (1978) found the segregation effect to be cumulative, with evidence accumulating over a period of a few seconds (see Fig. 2).

Cyclic sequences of greater timbral complexity have been also been used. Bregman and Pinker (1978) used a sequence that alternated a single tone with a pair of tones to reveal a trade-off between onset asynchrony and frequency separation in streaming: constituents of synchronous tone pairs are more difficult to capture into a competing stream than asynchronous pairs. Bregman et al. (1990) put into opposition streaming-by-fundamental and streaming-by-timbre, demonstrating the efficacy of both factors, albeit with a stronger effect of the fundamental. However, recent experiments with tones defined by unresolved high harmonics show that spectral shape can have an effect on streaming stronger than that of fundamental frequency, as discussed below (Vliegen et al., 1999).

Rogers and Bregman (1993) discuss three alternative explanations of the streaming effect. A fourth, the peripheral channeling interpretation of Hartman and Johnson (1991), is described below. Rogers and Bregman contrast Bregman's (1990) auditory scene analysis account, which favors sequential grouping by the Gestalt principle of frequency proximity, with those of van Noorden (1975) and Jones (1976). Jones proposed a theory based on rule-based predictability of sequences, while van Noorden suggested that hypothetical frequency jump detectors become adapted and are unable to follow the alternating pattern of tones.

Rogers and Bregman attempted to distinguish between the three accounts by measuring the effect of preceding "induction" tones on the streaming of a test sequence. All induction conditions led to an improvement in streaming effectiveness in comparison to a control condition which used low-intensity white noise. All induction sequences consisted solely of high frequency tones, ruling out van Noorden's proposed adaptation of frequency jump detectors. Induction sequences which differed only in the predictability of inducer tones performed no better than those containing irregular patterns of tones, in contrast to the predictions of Jones' theory.

A second experiment, using inducer sequences which varied in number and total duration of tone elements, demonstrated that segregation improved

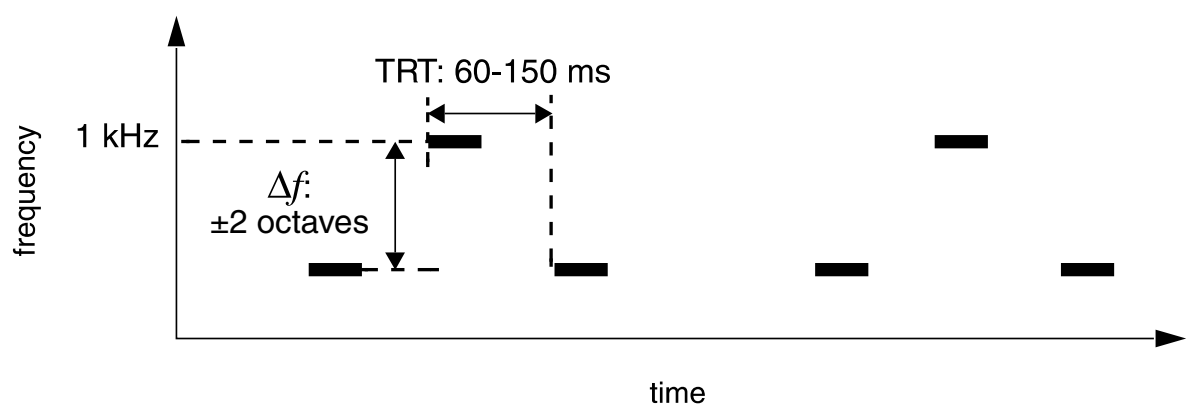

Fig. 2. Stimulus configuration for the streaming experiments of van Noorden (1975). The sequences of alternating sinusoidal signals are presented with differing frequency separations $(\Delta f)$ between the tones, and differing repetition periods (tone repetition time or TRT). 
with the total number of tone onsets rather than the summed tone durations in the inducer sequence. This finding runs counter to Bregman's original hypothesis that the inducer would set up a cumulative frequency bias for the higher tone, but was interpreted by Roger and Bregman as an example of sequential grouping by similarity of the number of tone onsets in inducer and test sequences.

Stream segregation has also been demonstrated using non-cyclic sequences. Deutsch (1975) used musical scales to demonstrate the dominance of grouping by frequency proximity over ear of presentation, while Hartman and Johnson (1991) asked listeners to identify pairs of melodies whose notes had been interleaved (Dowling, 1973). Hartman and Johnson investigated the idea that streaming could be explained purely by "peripheral channeling", that is, that streaming was promoted by manipulations that shifted streams into separate channels in the periphery, either spectral or spatial. Other factors, such as loudness and duration that could have distinguished between the interleaved streams but which did not lead to differentiation in peripheral channels, gave little advantage in segregating the melodies, thus supporting the peripheral channeling hypothesis. However, recent work on sequences of filtered tones carrying different pitches through the same high-frequency cochlear channels (within which they are unresolved) shows streaming effects that must rely on more centrally derived properties (Vliegen and Oxenham, 1999). Directly comparing these sequences (whose $f_{0}$ varies under a constant average spectrum) with sequences in which the spectrum is varied while holding $f_{0}$ constant, showed that both variations would impair the ability of listeners to judge the relative timing between tones - a characteristic effect of separation into different streams (Vliegen et al., 1999). However, consistent with the findings of Hartman and Johnson, spectral modifications seemed to have a stronger effect.

\subsection{Models}

A number of models which seek to explain streaming as an emergent consequence of early, low-level, auditory computations have been built, starting with the simple excitation-integration model of Beauvois and Meddis $(1991,1996)$. They sought to explain the perceptual coherence of tone sequences alternating in frequency, as used by van Noorden (1975), noting that listeners tend to hear more than one stream if the tone repetition time is sufficiently short, or if the frequency separation of the tones is sufficiently large. Beauvois and Meddis addressed these findings with a three-channel model, with bandpass channels centered at each of the tone frequencies and at their geometric mean. Noise was added to the rectified output of each channel, which was then averaged with a leaky integrator. The channel with the highest output was considered 'dominant', and activity in the other two channels was attenuated by $50 \%$. Temporal coherence was defined as the case when both flanking channels had similar average outputs; streaming was indicated if just one of these two channels dominated. The model exhibited temporal coherence if the tones were close enough in frequency for the central channel to dominate, or if the repetition rate was slow enough for each flanking channel to decay sufficiently and allow dominance to switch alternately between high and low bands. For faster rates, the internal noise would allow just one of the flanking channels to achieve and maintain dominance, indicating streaming; the boundary rate at which this occurred could be varied by changing the noise level. Beauvois and Meddis demonstrate that a single setting of this parameter allows the model to explain grouping by frequency and temporal proximity, as well as the build-up of streaming over time (Anstis and Saida, 1985). However, they acknowledge that the model cannot explain acrosschannel grouping phenomena such as that of Bregman and Pinker (1978).

McCabe and Denham (1997) extended the Beauvois and Meddis model to include multichannel processing and inhibitory feedback signals, whose strength they related to frequency proximity in the input. This mechanism leads to the suppression of stimulus components different from those responsible for the suppression. This residual activity is processed in a separate 'background' map, which in turn has the potential to 
inhibit components in the foreground map. McCabe and Denham (1997) suggest that their model can be viewed as an implementation of Bregman's old-plus-new heuristic, in which 'new' organization appears in the residual left after subtraction of 'old' components, based on the assumption of continuity. In addition to the streaming data accounted for by Beauvois and Meddis, their model caters for the influence of organization in the background on the perception of the foreground as found by Bregman and Rudnicky (1975). Vliegen and Oxenham (1999) observe that, being based on peripheral excitation patterns, neither of the models described above can account for their demonstration of streaming for spectrally matched signals with different $f_{0}$ s.

Most of the streaming mechanisms described above require cyclic repetition in order to produce a correlate of fission or fusion. An exception is the model of Godsmark and Brown (1999), which is based on maintaining multiple grouping hypotheses until sufficient information arrives to disambiguate potential organizations. Consequently, their model can handle a wide range of streaming phenomena including context-dependent and retroactive effects (Bregman, 1990). The approach taken by Godsmark and Brown involves training the model to produce streaming effects observed in simple tonal configurations, then observing the more complex emergent grouping behavior on stimuli such as polyphonic music. For example, the model produced good matches to listeners' performance in the interleaved melody identification task of Hartman and Johnson (1991).

\subsection{Discussion}

\subsubsection{Fusion and streaming}

We have taken streaming as the starting point for our discussion of auditory organization. However, the construction of streams presupposes the formation of distinct 'events', possibly requiring the fusion of energy in multiple frequency bands. Indeed, Bregman and Pinker (1978) set up competition between the formation of single events by the fusion of simultaneous tones, and the capture of one of the tones into a separate sequential stream. Factors governing fusion, such as har- monic relations and synchronous onset, have been further investigated and modeled through doublevowel stimuli, as discussed in the next section.

\subsubsection{The relevance of streaming phenomena to speech organization}

Cyclically repeated tonal configurations are hardly typical of the sound mixtures encountered by listeners. Consequently, it may be unwise to make inferences about the perceptual organization of everyday signals such as speech on the basis of streaming experiments. Bregman's rationale for the use of cyclic sequences (Bregman, 1990, p. 53)) is largely one of experimental pragmatism, and he urges the use of other methods to verify effects found using cyclic presentation. Since many explanations of listeners' responses to repeated stimuli would be difficult to apply to the general problem of auditory organization, it is conceivable that different mechanisms are invoked to those which apply in more natural settings.

An alternative way to explore grouping is to use stimuli that are somewhat closer to those present in a listener's environment, yet sufficiently simple to be controllable in an experimental setting. Double vowels are single-presentation stimuli which satisfy these constraints, and the next section looks at their perceptual organization and at models which attempt to account for listeners' identification performance.

\section{Double vowels}

\subsection{Listeners}

The finding that listeners are able to recognize simultaneously presented synthetic vowels at levels well above chance (Scheffers, 1983) has led to a large number of perceptual studies utilizing this socalled double-vowel or concurrent-vowel paradigm. Part of the attraction comes from the ease with which stimulus manipulations thought to promote perceptual organization can be performed on vowel pairs. For example, constituent vowels can be synthesized with different fundamental frequencies, modes of excitation, relative intensities and interaural time or level differences. 
In the 'standard' double-vowel experiment, listeners have to identify both constituents of synthetic concurrent vowel pairs (usually drawn from a set of five) of a given duration (typically $200 \mathrm{~ms}$ ). Key findings for a variety of double-vowel manipulations are:

- Concurrent vowels synthesized with the same $f_{0}$ can be identified at a level well above chance (Lea, 1992). When the choice is between five vowels, a typical result is correct identification of both constituents in $55 \%$ of trials.

- Pairs of whispered vowels are identified at about the same rate as vowels with a common $f_{0}$ (Scheffers, 1983; Lea, 1992). Whispered vowels may be constructed to contain minimal grouping cues, so performance in this task is usually taken as the baseline upon which improvements due to grouping are made.

- A difference in fundamental frequency between pairs of concurrent vowels leads to an absolute improvement of $10-15 \%$ in vowel identification performance, the effect beginning at a difference as small as a quarter of a semitone and asymptoting by 2 semitones. This basic finding of Scheffers (1983) has been replicated by several researchers (Assmann and Summerfield, 1990; Culling and Darwin, 1993; Lea, 1992; Meddis and Hewitt, 1992; de Cheveigné et al., 1997a).

- A difference in mode of excitation (voiced/whispered) between the constituent vowels leads to an identification improvement of around 10\% (Lea, 1992). Further, the whispered constituent of a voiced/whispered vowel pair was identified significantly more accurately than when both vowels were whispered, but the voiced component was no more intelligible than when both vowels were voiced and on the same $f_{0}$ (Lea, 1992).

- Identification performance varies with the harmonicity or inharmonicity of vowel pair constituents (de Cheveigné et al., 1997b). An inharmonic target vowel presented $15 \mathrm{~dB}$ below a harmonic masker vowel was significantly better identified than a harmonic target behind a stronger inharmonic masker.

- Swapping formants between the vowels, so that each $f_{0}$ carries the $\mathrm{F} 1$ of one vowel with the higher formants of the other, allows listeners to achieve the same improvement as in the standard condition up to an $f_{0}$ difference of 0.5 semitones (Culling and Darwin, 1993). Applying the $f_{0}$ difference only to the F1s of the vowels had a similar effect. Culling hypothesized that listeners used the time-varying excitation pattern caused by beating in the $\mathrm{F} 1$ region to identify constituents at times favorable to one or other vowel (Culling and Darwin, 1994), although this scheme has recently been called into question (de Cheveigné, 1999).

- Identification improvement with $f_{0}$ difference is smaller for brief $(50 \mathrm{~ms})$ stimuli than for longer (200 ms) stimuli (Assmann and Summerfield, 1990). Repeating the same $50 \mathrm{~ms}$ segment four times with $100 \mathrm{~ms}$ silent intervals did not lead to any improvement, but performance did improve when successive $50 \mathrm{~ms}$ segments were presented with the same silent intervals (Assmann and Summerfield, 1994). Some of this improvement was attributed to waveform interactions which allow better glimpses of one or other vowel at different times, but de Cheveigné (1999) presents results for vowels with extremely small differences in $f_{0}$ which argue against the glimpsing hypothesis, since the slow change in relative phase between such close frequencies does not provide for a significant variation in glimpsing conditions during the stimulus.

- One vowel of the pair (the 'dominant' vowel) can be identified at near $100 \%$ accuracy for stimuli as short as one pitch period, while identification of the non-dominant vowel improves with an increasing number of pitch periods (McKeown and Patterson, 1995). Introducing a difference in $f_{0}$ reduces the number of pitch periods required to reach maximum performance. As well as showing a clear effect of stimulus duration on identification of the non-dominant vowel, these results suggest that $f_{0}$ differences are not required for identification of the dominant vowel. The dominance effect can be removed by adjusting levels of constituents in each pair (de Cheveigné et al., 1995), a manipulation which may be necessary to allow the conditions of interest to surface.

- Shackleton and Meddis (1992) found that spatial separation of vowels resulted in no increase 
in identification performance for vowels with the same $f_{0}$ s. For different $f_{0} \mathrm{~s}$, spatial separation led to a small improvement.

- In a simulated reverberant environment, Culling et al. (1994) explored the robustness of binaural and $f_{0}$ difference cues, concluding that $f_{0}$ continued to be useful in reverberant fields that had removed the benefits of interaural timing information.

- Culling and Summerfield (1995b) used a reduced form of double-vowel stimulus, in which each vowel was represented by two noise bands, to demonstrate an absence of across-frequency grouping by common interaural delay. They went on to show that introducing an interaural decorrelation (as opposed to a delay) improved identification of the vowels.

- No effects of common frequency modulation on double-vowel identification have been found (Darwin and Culling, 1990; Culling and Summerfield, 1995a).

Reviews of concurrent vowel segregation can be found in Lea (1992), de Cheveigné (1993), Summerfield and Culling (1995) and de Cheveigné et al. (1995).

Taken together, these findings suggest that listeners identify double vowels via a variety of stimulus properties conveyed by the detailed timefrequency structure of the auditory response. Some of these can be cast as cues for primitive perceptual grouping, but the role of factors which enable the engagement of presumed vowel templates or schema (e.g. locally favorable target-tobackground level; see (Assmann and Summerfield, in press)) needs to be carefully assessed. In fact, no firm conclusions about mechanisms can be drawn at present, although a number of detailed proposals have been made. These are discussed below.

\subsection{Models}

The first computational model of double-vowel segregation was constructed by Scheffers (1983) himself. Scheffers' model employed a harmonic sieve algorithm (Duifhuis et al., 1982) in which each $f_{0}$ estimate generated a sequence of frequency intervals around each harmonic frequency for that $f_{0}$. Peaks in the excitation pattern of the stimulus which fall through these sieve intervals contribute to the evidence for that $f_{0}$, and the $f_{0}$ with the largest weight of evidence is chosen. Scheffers developed an algorithm which finds the pair of $f_{0} \mathrm{~s}$ which together account for the greatest proportion of peaks in the excitation pattern. His model consistently underperformed listeners (e.g. for $\Delta f_{0}=0$ the model correctly identified both vowels in $27 \%$ of cases whereas listeners manage $45 \%$ ), but showed a small improvement with a $\Delta f_{0}$ of 1 semitone (38\% versus $62 \%$ for listeners). However, this improvement disappeared at four semitones difference $(27 \%)$ while listeners' performance remained at $62 \%$.

Since Scheffers' harmonic sieve model operates on intensity peaks in an estimate of the cochlea excitation pattern, we consider it a 'place' model even though the pattern could be derived by temporal processing, the hallmark of a 'time' model. Conventional 'time' models compute correlates of $f_{0}$ in the time domain typically by autocorrelation: if the time-domain processing is applied to signals derived from an earlier spectral analysis, the model is termed 'place-time'. Place, place-time and puretime models for double-vowel pitch estimation and segregation are discussed in (de Cheveigné, 1993).

Autocorrelation is particularly useful for the detection of periodicity. Several different autocorrelation-like models have been proposed for auditory computation. In 1951, Licklider suggested a structure for periodicity detection consisting of a series of delays; delayed versions of the signal were combined with undelayed signal in a multiplier and averaged in an integrator. The series of delay elements thus maps out uniformly increasing delays, and the model output at any place along this delay axis represents a running autocorrelation with the lag given by the total delay applied to the signal in that channel.

Assmann and Summerfield (1990) compared two models on the concurrent vowel segregation task. One was a place model similar to that used by Scheffers. The other involved a place-time analysis based on detecting periodicities using an autocorrelation of the output at each channel of a periphery model. Their place model estimated vowel spectra by sampling the excitation pattern at harmonics of the $f_{0}$ s found by their implementation of 
Scheffers' sieve. The place-time model estimated vowel fundamental $f_{0}$ s by finding the two largest peaks in a "summary autocorrelation" function created by summing individual autocorrelation functions across channels. Fig. 3 depicts an autocorrelogram of a vowel pair together with its summary. Vowel spectra were then estimated by taking slices through the autocorrelation functions at lags corresponding to the two pitches. Assmann and Summerfield evaluated the performance of the place and place-time models (and a variant that preceded autocorrelation with a non-linear compression modeling the cochlea's inner hair cells) and found that the place-time model came much closer to accounting for listeners' performance on the same task.
Meddis and Hewitt (1992) also used an autocorrelogram analysis, but chose a different segregation strategy. They first determined the lag of the largest peak in the summary autocorrelogram, then they selected those channels whose individual autocorrelation functions possessed a large peak at this lag as belonging to the 'dominant' voice; the remaining channels were deemed to belong to the other voice. Meddis and Hewitt then automatically classified each vowel based on the short-lag autocorrelation values for summary autocorrelations based on each subset. (Since the autocorrelation at very short lags - the "timbre region" - characterizes the waveform at time scales below the pitch cycle, it is well correlated with vowel identity.) Their vowel recognition results were very close to

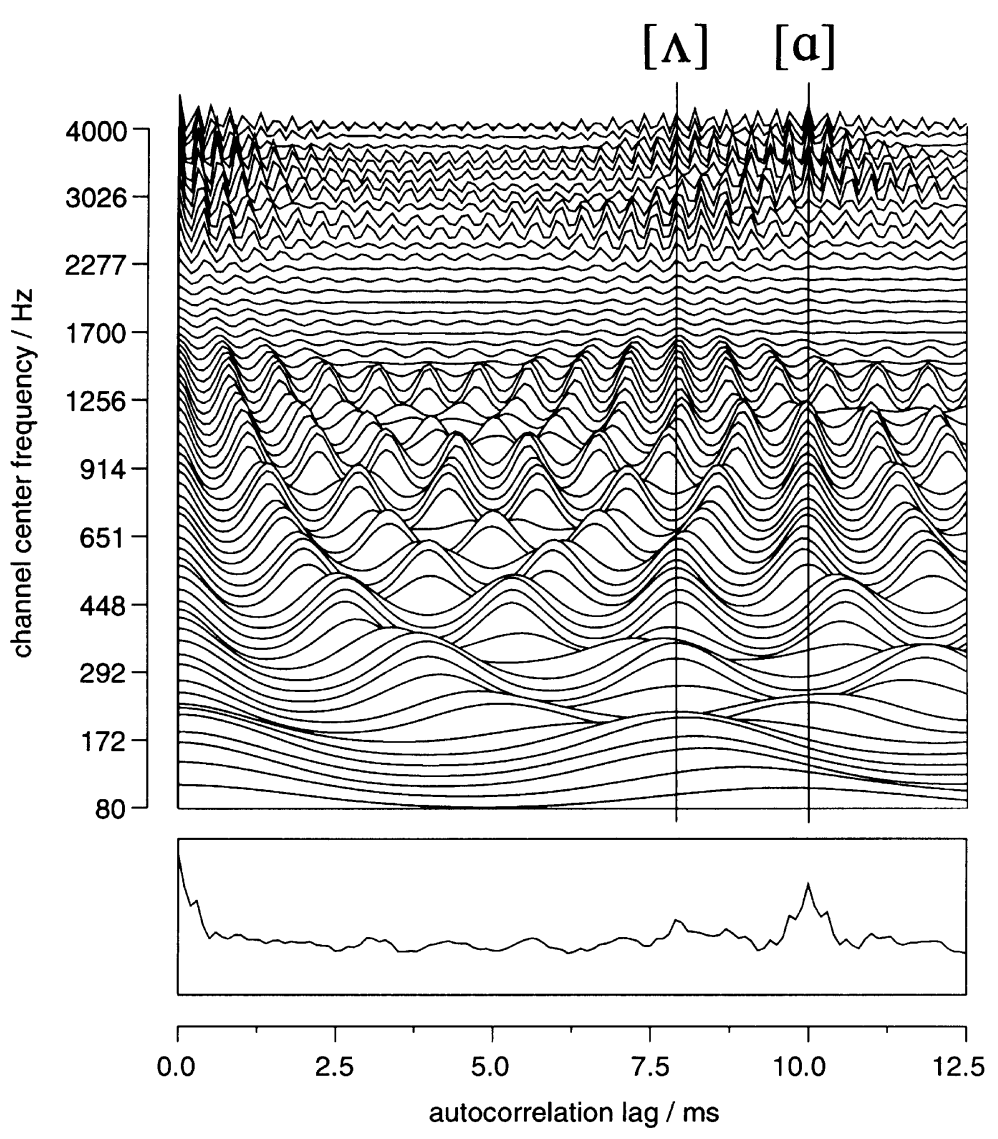

Fig. 3. Autocorrelogram of a synthetic double-vowel pair $[\Lambda]$ on a fundamental of $126 \mathrm{~Hz}$ and [a] with a fundamental of $100 \mathrm{~Hz}$ ). The summary correlogram (lower panel) shows a strong peak at an autocorrelation lag of $10 \mathrm{~ms}$, corresponding to periodicities in the signal at harmonics of $100 \mathrm{~Hz}$. A smaller peak at $7.9 \mathrm{~ms}$ corresponds to harmonics of $126 \mathrm{~Hz}$. 
the results of subjective tests performed by Assmann and Summerfield. A weakness of the Meddis and Hewitt model is that it cannot account for separation, observed in listeners, when the entire spectrum is dominated by one vowel (de Cheveigné et al., 1997a; de Cheveigné, 1999), since no autocorrelogram channels are allocated to the weaker vowel.

More recently, Berthommier and Meyer (1997) have shown how amplitude modulation (AM) information can be used as a basis for double-vowel segregation. They computed an AM map by taking the envelope from each of a bank of auditory filter outputs and performing a spectral analysis giving results in the pitch range. The AM map conveys envelope modulation information as a function of cochleotopic frequency, and can be used to group channels possessing a peak at the same modulation frequency. However, Berthommier and Meyer note that the presence of harmonics in the AM spectrum can cause spurious peaks, and propose a further transformation using a harmonic sieve to group these harmonics together prior to vowel classification.

One issue which has been explored with the aid of double-vowel stimuli is the question of whether listeners exploit the periodicity of the target vowel to enhance or select that vowel, or whether the $f_{0}$ of the interfering vowel is used to attenuate or cancel it - or indeed whether a combination of both strategies is used. An $f_{0}$-based enhancement strategy is advantageous when the target signal is periodic and dominant, since $f_{0}$ estimates will be more accurate. Conversely, cancellation ought to favor situations with a strong periodic interfering sound.

A number of authors have considered this question in detail (Lea, 1992; de Cheveigné, 1993, 1997). Lea argued that an enhancement mechanism should favor target vowels that were voiced rather than whispered, regardless of the masker. By contrast, a cancellation model predicts that any kind of target is easier to pick out if the masking interference is voiced. Lea's experimental results support cancellation by suggesting that listeners can exploit the periodicity of an interfering vowel to help identify a target sound, but that they cannot use target periodicity to extract a vowel from a mix. de Cheveigné (1993) proposed a time-domain cancellation model exploiting the property of comb filters to produce zero output for a periodic input whose period matches the filter's lag coefficient. It is necessary to know the lag parameter in order to effect the cancellation, but this can be found by searching in filter lag space for a minimum output. de Cheveigné tested a neural-style implementation by feeding it auditory nerve responses to concurrent vowel stimuli (Palmer, 1990) and demonstrated that it could successfully isolate the periodicities of either vowel. He later showed that the model could account accurately for listeners' responses in a double-vowel experiment (de Cheveigné, 1997). de Cheveigné (1993) also suggested using a cascade of two comb filters to estimate the fundamental frequencies of both concurrent voices. He compared the scheme with the Assmann and Summerfield (1990) technique of choosing the two largest peaks in the summary autocorrelogram. Using voiced tokens of natural speech, and based on a criterion of the percentage of estimates falling further than $3 \%$ away from the correct $f_{0}$, he found that the comb filter cascade scheme resulted in $10 \%$ errors, while the summary correlogram method was in error in $62 \%$ of cases.

\subsection{Discussion}

\subsubsection{Interplay between pitch and grouping}

One issue highlighted by models of double-vowel segregation is the interplay between grouping and pitch: does grouping depend on pitch identification, does grouping determine pitch, or do they both influence each other? It is known, for instance, that onset asynchronies among partials of a tonal complex can influence pitch (Darwin and Ciocca, 1992). The very different models of Meddis and Hewitt (1992) and de Cheveigné (1993, 1997) both rely on an initial pitch determination. For Meddis and Hewitt, this allows the grouping of channels, but the weaker pitch is based on the excluded channels, thereby introducing a mutual dependence of pitch and grouping.

\subsubsection{The time course of double-vowel segregation \\ Models of double-vowel segregation typically operate over short time windows and have diffi-}


culty accounting for perceptual findings of variations in double-vowel perception that depend on wider temporal contexts such as the duration of the stimuli (e.g. the results of Assmann and Summerfield, 1994; McKeown and Patterson, 1995). Culling and Darwin (1994) were able to explain listeners' double-vowel identification for $f_{0}$ differences below a quarter semitone without using autocorrelation. Their model used a temporally smoothed excitation pattern as input to a singlelayer perceptron trained to recognize one of five vowels, and demonstrated an increase in identification with increasing $f_{0}$. They attributed this result to the possibility of glimpsing the changing spectrum arising from the low-frequency beating caused by the small $f_{0}$ difference. These results are considered further in the discussion of extending cues across time in the next section.

\section{Accumulating grouping information across time}

In this section we consider how the auditory system combines information received at different times for the purpose of organization. It is easy to recognize a temporal aspect to grouping in "buildup" phenomena (such as those discussed above in relation to streaming) where the organization of a stimulus depends on its duration. Many of these phenomena might be explained simply as sluggishness in the calculation of low-level features, but some may require a separate, central process for integrating a grouping attribute that is based on several cues. We now examine some of the evidence for this type of mechanism.

\subsection{Listeners}

The double-vowel paradigm combined sounds whose spectrum and period repeated exactly every pitch cycle, and in this respect they are unlike most real-world sounds for which coordinated acrossspectrum changes offer a powerful indication of common origin. In the description of grouping presented by Bregman (1990), individual sound elements such as harmonics are grouped into sources on the basis of various cues. Implicit in this account is a central reckoning in which each element is tracked over its period of existence, and evidence for grouping is gathered, stored and applied over the whole element - even though that evidence may arise from a limited time interval.

\subsubsection{Extending a single cue across time}

A single cue may influence grouping at times remote from its own temporal focus. Thus, although onset information is present only at the beginning of a tone, the segregation of a harmonic that starts $40-80 \mathrm{~ms}$ before the rest of a cluster will persist for many hundreds of milliseconds - as judged from its contribution to timbre (Darwin, 1984) or pitch (Moore et al., 1986). Thus, a single cue can exert an influence long after it has occurred.

An equally important role for time in low-level grouping stems from the possibility that certain cues need a significant signal duration for their determination. An accurate pitch judgment may require averaging over time to reduce internal noise. This may contribute to McKeown and Patterson's (1995) observation of increasing perceptual delay in the organization of mixtures as their pitch separation decreases. Other cues are intrinsically dependent on time, such as the detection of cyclic repetition in iterated frozen-noise stimuli (Guttman and Julesz, 1963; Kaernbach, 1992). Another example, described in (Mellinger, 1991), is the Reynolds-McAdams oboe signal in which a small degree of frequency modulation is applied only to the even harmonics of a signal that initially has the character of an oboe, but subsequently splits into a clarinet-like tone (formed from the unmodulated odd harmonics) and something like a soprano at an octave above (corresponding to the modulated harmonics). The listener may require several hundred milliseconds of observation before the frequency modulation can be reliably recognized and used to separate the sound into two percepts, but once the threshold has been reached the influence is much like an instantaneous cue in that it applies immediately to the tracked continuations of the sound.

Mistuning in double-vowel segregation and harmonic clusters provides an interesting case. In both situations, identification (of the different vowels, or of the presence of a mistuned harmonic) 
becomes more difficult as the signal duration is reduced from 200 to $50 \mathrm{~ms}$ for vowels (Assmann and Summerfield, 1994) or 400 to $50 \mathrm{~ms}$ for harmonics (Moore et al., 1986). This suggests a timeintegration process able to make finer distinctions when given more of the signal. The alternative explanation, proposed by Culling and Darwin (1994) is that phase interactions between slightly mistuned harmonics give rise to 'beating' modulations in both kinds of stimuli. This may be a cue to discrimination in itself, or it may offer 'glimpses' - moments when signal interactions make the identification task briefly much easier. A longer stimulus has a greater chance of spanning such a glimpse, producing better identification on average. If the benefits of glimpsing relied solely on the single best glimpse, a shorter stimulus that happened to contain a glimpse would be equally well segregated. This is partially supported by the result that certain $50 \mathrm{~ms}$ segments give better identification scores than others (Assmann and Summerfield, 1994). However, in that study no $50 \mathrm{~ms}$ segment allowed the level of discrimination that occurred with the $200 \mathrm{~ms}$ segments, suggesting a benefit from temporal integration available only in the longer stimuli.

Glimpsing has also been proposed to explain the phenomenon of comodulation masking release (CMR), in which the threshold for a sinusoidal target beneath a narrow-band noise masker can be reduced by adding noise bands separate from the target/masker band - if the added bands share the amplitude-modulation envelope of the on-band masker (Hall et al., 1984). Although several possible mechanisms have been indicated (Schooneveldt and Moore, 1989), at least some of the effect appears to result from a comparison between the envelopes in the on-band and flanking frequency channels. For instance, the auditory system could monitor the flanking noise envelopes to detect instants when the on-band masker was briefly at a very low amplitude, giving the most favorable opportunity for 'glimpsing' the target tone, or it could apply processing similar to Durlach's, 1963 equalization-cancellation (EC) model (Buus, 1985) to detect small differences between the envelopes. A prior auditory process would be required to confirm that the noise bands are co- modulated and deserve to be compared. Such a process probably involves integration along time of repeated synchrony between detailed signal features such as amplitude peaks, or a more direct calculation of the running cross-correlation (Richards, 1987).

In these examples, temporal integration relates to a single cue only, and thus no separate grouping property is required - the integration can be a direct part of the cue calculation, and the grouping could be rigidly determined on the basis of the single strongest cue. By contrast, the next section considers interactions between different cues, which point to more sophisticated grouping processes.

\subsubsection{Integrating different cues}

Combining different kinds of evidence is one of the most intriguing aspects of auditory organization, and experiments in cue competition form an important paradigm. As previously mentioned, Bregman and Pinker (1978) constructed stimuli that set in competition the fusion of approximately simultaneous sine tones and the streaming of sequential tones close in frequency. Other experiments have similarly varied onset asynchronies to investigate their influence on the grouping effects of mistuning (Darwin and Ciocca, 1992; Ciocca and Darwin, 1993) and spatial location (Hill and Darwin, 1993). In each case, one cue could be used to compensate for changes in the other, so for instance the increased contribution to a pitch percept of a harmonic as its mistuning falls to $3 \%$ could be reduced again by starting it $30 \mathrm{~ms}$ earlier than the rest of the complex. This suggests that, at some level, both cues are mapped to a single perceptual attribute and thereby become interchangeable.

In fact, the organization of all sounds involves the combination of different cues: any simple signal exhibits numerous attributes relevant to grouping such as common onset, harmonicity and common interaural properties. Although a given experiment typically investigates a single dimension while keeping constant other aspects of the signal, the overall organization will depend both on the varying and invariant properties. Thus the reduced threshold for detecting mistuned 
harmonics in longer signals could indicate the kind of integration-along-time discussed above, but it may also reflect a dynamic balance between a continuously present mistuning cue and the decaying influence of the onset cue. This is related to a demonstration by Pierce (1983), who constructed a harmonic complex with individual components that increased abruptly in level. At the moment of the change, the boosted harmonic is perceived as separate from the others, but over a time scale of seconds it will 'merge' back into the harmonic complex as the step-change in amplitude becomes increasingly remote in time, and the various tendencies to integrate simultaneous sounds regain dominance.

Many experiments have used onset manipulations to investigate the grouping aspects of harmonicity (Darwin and Ciocca, 1992), formants (Darwin, 1984) and lateralization (Woods and Colburn, 1992). The paradigm typically assumes that a degree of onset asynchrony can preemptively remove the contribution of a particular spectral region from the derived properties of the larger percept. To control the interaction between onset and other cues, the stimuli employed are typically very short; in contrast, the long stimuli of Pierce expose these interactions to the full.

The numerous factors influencing the integration of evidence derived from different processes are apparent in experiments concerning the segregation of speech on the scale of sentences. Brokx and Nooteboom (1982) used synthesized speech stimuli with a constant $f_{0}$ throughout an utterance (i.e. monotone pitch), and varied the frequency separation from monotone interfering speech. Unlike the double-vowel experiments, these complete utterances contained additional cues such as the common energy modulations within each voice, and higher-level linguistic-semantic constraints. This greater complexity reveals an interesting trend: whereas identification improvement of static double vowels has plateaued at $12 \%$ difference in $f_{0}$ (Assmann and Summerfield, 1990), Brokx and Nooteboom saw an approximately linear benefit of pitch separation on intelligibility out to a pitch difference of $20 \%$. More recent studies by Bird and Darwin (1998) have followed this trend out to $60 \%$ differences in $f_{0}$.

\subsection{Models}

Although the time dimension provides grouping mechanisms with extra information, it adds a great deal of complexity to the computational task when compared to the problem posed by double vowels. We will now look at some of the models that have dealt with these issues by emulating aspects of the organization performed by human listeners on sound scenes whose evolution is measured in seconds.

Weintraub (1985) described the first computational model explicitly motivated by experimental studies of auditory organization. His goal was to separate mixtures of two simultaneous voices, with a view to improving automatic speech recognition for each voice. His system used autocoincidence (a low-complexity version of autocorrelation) of simulated auditory nerve impulses to separate signals of different periodicities in peripheral frequency bands. Context dependence was included in the form of a Markov model tracking the states of each speaker as silent, voiced, unvoiced or transitional. The optimal labeling provided by this model controlled a dual-pitch tracking algorithm and guided the division of the signal energy into spectra for each of the two voices. Although the benefits of his system measured through speech recognition scores were equivocal, he prepared the ground for subsequent modeling work by identifying the problem of working solely from local features without the influence of top-down factors.

Cooke's (1991) system decomposed the acoustic mixture into a set of time-frequency tracks called "synchrony strands", then grouped these components using harmonicity (for the lower frequency resolved partials) and common amplitude modulation (for the unresolved harmonics in the upper spectrum). Harmonic grouping employed a temporally extended form of Scheffers' harmonic sieve, illustrated in Fig. 4. Since grouping relies on identifying each distinct element correctly, situations where features collide and cross can lead to catastrophic mislabelings if incorrect continuations are tracked after the collision. However, Cooke's algorithm can handle sounds with crossing fundamental frequency contours because attributes such as pitch are calculated after the 

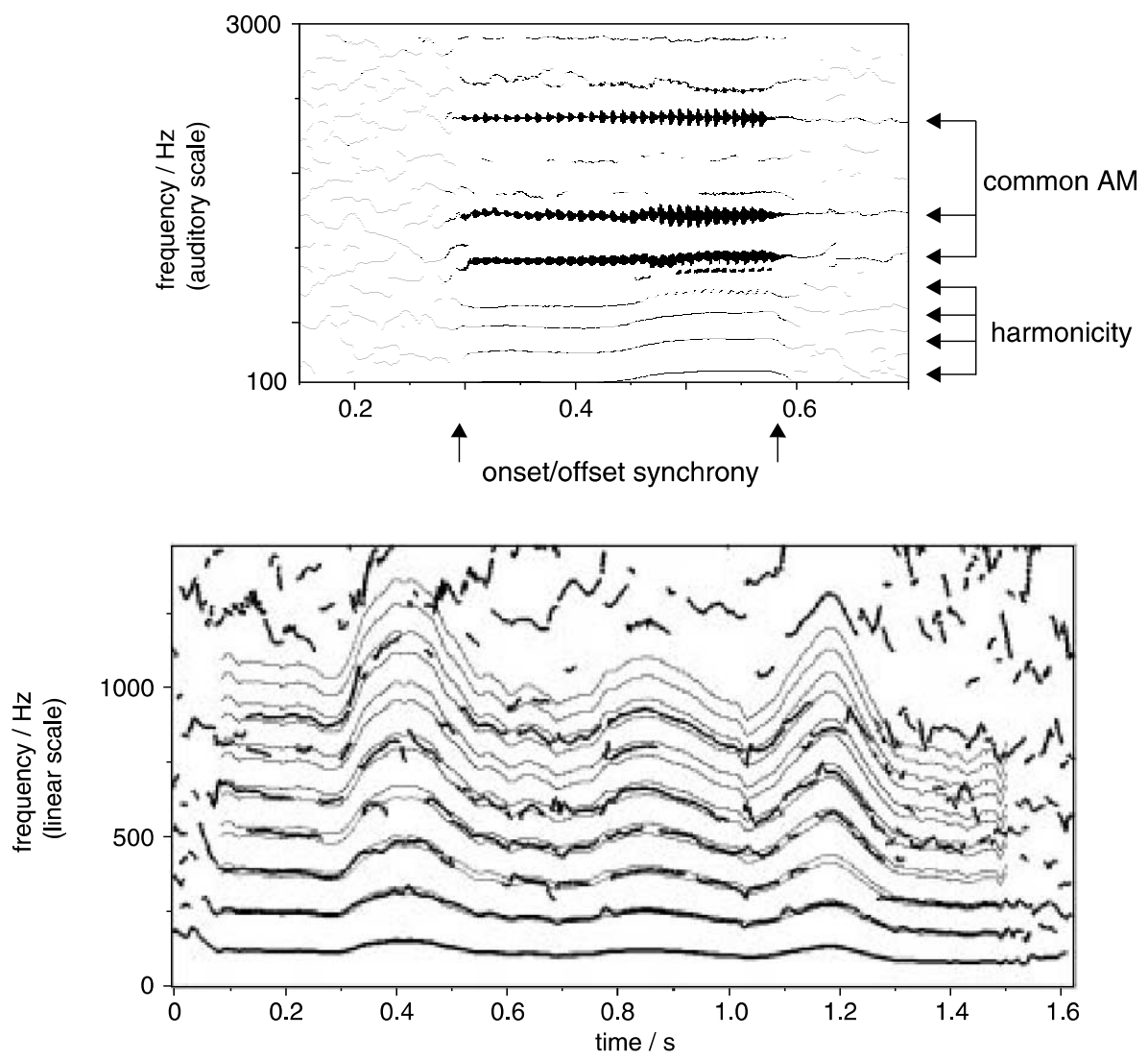

Fig. 4. Time-frequency representation and grouping cues used in (Cooke 1991; 1993). Upper: synchrony strands and grouping indications for a natural syllable. Strands corresponding to resolved harmonics are visible in the low-frequency region. In the mid-highfrequency region, strands represent formants F2-F4. The line width encodes instantaneous amplitude, and a clear pattern of amplitude modulation is visible. Lower: synchrony strand representation of the lower spectral region for a completely voiced utterance, overlaid by a time-frequency harmonic sieve (thin lines). Strands which fall between pairs of sieve lines are deemed to belong to the same source.

tracking of partials, themselves less likely to manifest crossing due to the local spectral dominance of one or other source. A further benefit is that the likelihood of a partial falling into an incorrect sieve 'groove' decreases rapidly as the sieve extends across multiple time steps. To illustrate the generality of the approach, Cooke's model was tested on 100 mixtures of sentence material combined with other acoustic sources including other sentences. In all cases, substantial improvements in signal-to-noise ratio resulted, although, as discussed in Section 7, it is not clear quite how to interpret such figures.

Similar considerations motivated Brown and Cooke, 1994 in his study of musical separation.
His model tracked spectral peaks across time, grouping peaks with similar onset times or common frequency modulation. Mellinger's system, like real listeners, maintained an evolving organization, in contrast to Cooke's approach which left all processing until the end of the signal. Newly detected harmonics had a fixed 'grace period' to build up affinity with existing harmonics, after which they were added to an existing group, or used as the basis for a new group. Mellinger used the Reynolds-McAdams oboe as one of his test signals; the sudden change in perception from one to two sources experienced in that sound is reflected in an abrupt change in his model's organization, when the initial single source loses the even 
harmonics to a newly spawned group (corresponding to the soprano) which has a greater internal coherence of frequency modulation.

Brown (1992) (see also (Brown and Cooke, 1994)) also used a decomposition into partials, and introduced two further innovations. First, he computed a local pitch for each partial by combining the summary autocorrelation function (see Fig. 3 of the previous section) with the local autocorrelation function in the spectral region of the partial. This has the effect of emphasizing the relevant pitch peak in the summary, which is used to define the underlying pitch contour for each partial. Second, Brown employed a tonotopically organized computational map of frequency movement to predict the local movement of partials. His system searched for groups of elements with common pitch contours, favoring sets with common onset times. Brown compared this approach to that obtained using frame-by-frame autocorrelation-based segregation and found that the use of temporal context produced a substantially larger improvement in SNR for the target sentence in a mixture.

\subsection{Discussion}

\subsubsection{Defining an element}

The outline of the auditory organization process underlying nearly all work in the field involves an analysis of the sound signal into basic elements, defined by their locally coherent properties, from which grouping cues may be calculated and for which grouping decisions can be made. In simple experimental stimuli built from sine tones and regular noise bursts, defining the boundaries and extent of the elements is usually unambiguous. Unfortunately, this is not the case for the noisy, complex sound scenes encountered in the real world. Modelers have often dealt with this problem by limiting their elements to be those defined by strong spectral peaks, but the ability of listeners to organize all kinds of signals, with or without strong spectral energy concentrations, may demand a more comprehensive approach. Recent modeling work has attempted to cover a wider range of sounds. Ellis (1996) suggests that a simple vocabulary of tonal, noisy and impulsive elements may encompass most perceptually salient signals, and Nakatani et al. (1997) present a detailed ontology of the signal attributes that characterize different classes of sound such as speech and music. However, to analyze a particular signal into these more complex elements is difficult and frequently gives ambiguous results.

\subsubsection{Different groupings for different attributes?}

Darwin and Carlyon (1995) have cautioned that grouping should not be considered an "all-ornone" process. Certainly, the interaction of cues in grouping makes it misleading to search for a single threshold at which a feature such as mistuning or asynchrony will lead to segregation. These thresholds depend on the contributions of the other cues in a particular experimental paradigm. However, the deeper point relates to results where measurements for a single stimulus continuum give different grouping boundaries when they are based on different attributes. Thus, when a resolved harmonic is mistuned relative to the others in a complex, subjects perceive the harmonic as distinct for detunings of $2 \%$; however, it continues to have an influence on the pitch they perceive for the remaining complex out to mistunings of $8 \%$ or more (Moore et al., 1985). Darwin and Carlyon see this as evidence for separate grouping processes simultaneously at play - one for the perception of the number of sources, and a different one for the calculation of pitch. Alternatively, the pitch calculation mechanism, even when attempting to exclude a spectral region from a particular percept, might still allow some influence to 'spill over', i.e. there may be a limit to how completely a particular harmonic can be removed from a pitch calculation simply due to grouping effects. This explanation is at odds, however, with Ciocca and Darwin's (1993) results that a sufficiently large onset time difference can completely remove a harmonic. Their experimental design further demonstrated that this was a grouping phenomena rather than being a result of another effect such as adaptation or fatigue.

\subsubsection{Expectation as the mechanism for combining information along time}

This section has considered the ways in which the properties of individual elements may govern 
their mutual grouping. However, the grouping process may be influenced by properties that belong not to single elements, but those that arise from the conjunction of several elements. We can consider these influences as "expectations", or short-term biases towards particular interpretations. For instance, in the experiments of Hukin and Darwin (1995), a stream of captor tones preceding a harmonic complex was able to reduce the influence of one harmonic. The captor tones set up an expectation that a similar tone in the complex belonged to them rather than to the complex.

This section has mainly assumed that properties of an element at one time (e.g. its onset asynchrony) can affect its treatment at later times, but we now see that associating grouping effects with specific elements is too narrow a perspective: The grouping effects of the captor extend into later disconnected elements. Thus perhaps onset asynchrony, rather than marking specific harmonics as distinct, sets up a more diffuse expectation that affects those harmonics yet does not depend on the direct physical continuity between the onset part of the signal and the subsequent harmonic. Although this distinction may be largely academic if the alternatives cannot be differentiated, it raises questions of how expectations are represented, and how they exert their influence. The following section considers in more detail the action of influences which we consider 'top-down' because an abstract property affects a more concrete percept.

\section{Context, expectations and speech}

Our detailed perceptions of the world often turn out to be built up from very slender supplies of sensory information - such as the $2^{\circ}$ cone of highresolution image achieved by the fovea in the eye, or the occasional spectro-temporal glimpses of target speech in a noisy environment. We are able to operate with limited information in part because our perceptual system is extremely efficient at exploiting and integrating constraints concerning what we know to be the plausible alternatives in any given situation. The persistence of the physical world makes it unnecessary to scan continuously in order to have an accurate internal image of our surroundings (in most cases). Similarly, when listening to partially masked speech, our experience of what comprises a grammatically or semantically reasonable utterance may provide just enough information to construct an impression of how the original speech sounded. These aspects of cognitive function involving knowledge and expectation are poorly understood and difficult to research, yet they are of central importance to auditory perception.

Progress in automatic speech recognition in the last decade has been due in a large part to successful techniques for combining 'bottom-up' information derived from the input signal with 'top-down' constraints imposed by the recognizer's knowledge of vocabulary and grammar. Speech perception in humans similarly draws heavily on expectations to achieve perceptual organization. Later in this section, we will discuss some of the emerging work on integrating models of auditory scene analysis with speech recognition systems. First, we look at some of the experimental results demonstrating this principle in action.

\subsection{Listeners}

\subsubsection{Local context and "old-plus-new"}

An expectation is a state of the auditory processing system that will substantially affect the interpretation of a subsequent stimulus. As an example, consider the way in which listeners compensate for the spectral coloration imposed on a signal by the transmission channel. A simple filter can be constructed to convert the vowel sound in an utterance of "bit" so that, when heard alone, a listener will hear it as "bet" (Watkins, 1991). However, if the altered word is prefixed with a carrier phrase ("Please repeat the word: bit") also modified by the static coloration, the word is restored to its original phonetic identity. Through exposure to the longer sample, the auditory system has separated the effects of source speech and channel coloration, and has compensated for the latter in the interpretation of the target word. We would term this an expectation because in that it reflects the action of an abstracted property of the context (the inferred coloration) to alter the categorical perception of a 
concrete target signal, which is interpreted relative to that coloration. (Note that a similar effect from an adaptation mechanism, where each channel was normalized to remove slowly varying coloration, would not involve any high-level linguistic analysis of the context, and thus would not be covered by this definition.)

Expectation encompasses a general principle of auditory perception termed "old-plus-new" by Bregman (1990), relating to the powerful realworld constraint of independence among sound sources. Any abrupt change in the properties of the aggregate signal probably reflects a change in only one source (rather than coincidental changes of multiple sources), and a change in the total spectrum that consists of only an energy increment will be interpreted as the addition of a "new" source, with all the existing "old" sources continuing unchanged. The signal following the change is interpreted as being old-plus-new, and the properties of the new source are determined by finding the difference between the signal before and after the change.

The old-plus-new idea is illustrated in Fig. 5 (after (Bregman, 1990, p. 344)). The alternation between narrow and broader bands of noise is heard not as switching between two different signals but as a continuous low noise to which high noise bands (the difference between the narrow and the broad) are periodically added. Physically, the two interpretations are equally valid, but the auditory system irresistibly chooses division in frequency because it meets the old-plus-new crite- rion. The interpretation as the alternation between the two noise bands would require the less likely event of the narrow band of noise turning off at the very instant that the broader band turns on, although in practice we may well have constructed the signal that way.

\subsubsection{Continuity and induction}

The most dramatic consequences of expectations in the auditory system occur when an object or source is perceived in the absence of any direct local cues. In these situations, the perceived object is 'induced' from expectations set up by its context.

The simplest illustration of induction is the continuity illusion (Bregman, 1990, p. 28, studied earlier as the "pulsation threshold" e.g. in Houtgast, 1972 and in Thurlow and Elfner, 1959). If a steady tone has a brief burst of wide-band noise added to it, the energy of the noise may mask the tone, leaving the auditory system without direct evidence that the tone is present during the noise (indeed, for increasingly intense and/or brief noise bursts, it is impossible to say if a tone is present with any certainty a posteriori). In these circumstances, the percept is typically of the tone continuing during the noise despite the absence of tonal features from the stimulus during the burst. The auditory system rejects the interpretation that the tone has ceased during the noise burst since, although it is an adequate explanation of the stimulus, it violates the old-plus-new principle.

More complex examples of auditory induction are provided by the phonemic restoration

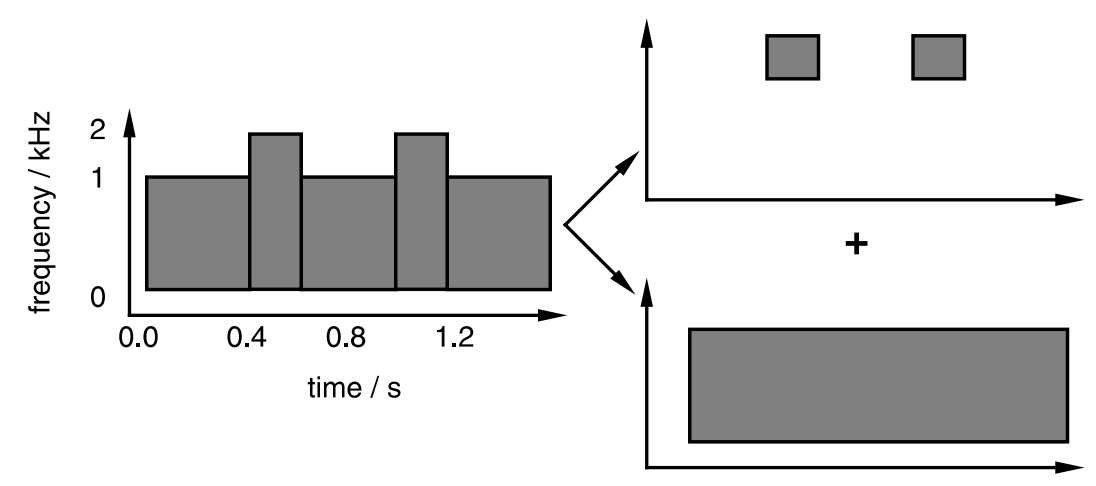

Fig. 5. The old-plus-new principle: schematic representation of the alternating narrow- and broad-band noise stimuli, and its perceptual organization. 
phenomena investigated by Warren (1970) and others. In the original demonstration, a single phoneme (the first /s/ in "legislatures") was attenuated to silence then masked by the addition of a cough. Not only were listeners unaware of the deleted phoneme (the speech was heard as complete), but they were unable to specify the exact timing of the cough, making a median error of five phonemes. Evidently, auditory processing had exploited the redundant information in the speech signal (coarticulatory, phonotactic and semantic) to induce the identity of the masked (missing) segment, a process so complete that, at the level of conscious introspection, it was indistinguishable from direct (non-restored) hearing. Subsequent experiments showed that a keyword occurring several syllables after the masked segment could provide the semantic constraint to restore the deleted phoneme, since listeners would reliably perceive different restorations for stimuli that differed only in the final keyword (Warren and Warren, 1970). These results demonstrate not only the very powerful effect of expectation in the perception of speech, but also that expectations can operate backwards in time. Induction also appears to operate between ears ("contralateral induction", Warren and Bashford, 1976) and across the spectrum ("spectral induction", Warren et al., 1997). In the latter study, the spectrum is reduced down to two narrow signal bands with a commensurate reduction in intelligibility. The introduction of an intervening spectral band of noise then modestly increases intelligibility.

Speech information can be combined across regions disjoint in both time and frequency, as demonstrated by "checkerboard noise" masking experiments of Howard-Jones and Rosen (1993). They used stimuli in which speech was alternated with noise in several frequency bands, such that half the bands carried unobstructed speech while masking noise was added to the interspersed remainder, and the pattern of noisy and clear channels flipped every $50 \mathrm{~ms}$ to give noise interference that resembled a checkerboard on a log-frequency spectrogram. They found that for a two-channel division (above and below $1.1 \mathrm{kHz}$ ), listeners were able to tolerate a level of checkerboard noise $10 \mathrm{~dB}$ higher than control conditions of noise gated in one channel but continuous in the other, demonstrating that information from separate frequency regions was being integrated across time. For wide-band pink noise gated at $10 \mathrm{~Hz}-$ i.e. simultaneous glimpses in high and low channels - a further $7 \mathrm{~dB}$ of SNR decrease was acceptable. Their result supports the notion of a central speech hypothesis (a further kind of expectation) that gathers information from any available source, rather than more local processes acting to integrate information only within frequency channels. There are numerous other unnatural manipulations of speech from which listeners recover intelligibility: see (Cooke and Green, in press; Assmann and Summerfield, in press) for further discussions.

\subsubsection{Speech as the best explanation}

The capacity to infer the presence and identity of speech with limited evidence is well demonstrated by sine-wave speech (Bailey et al., 1977; Remez et al., 1981, 1994), in which time-varying frequencies and levels of the first three or four speech formants are resynthesized as pure sinetones, removing cues to the excitation source. Although listeners hear sine-wave utterances as a combination of whistles (the interpretation that might be expected), they are often able to interpret them as speech, particularly when so instructed.

The combined perception of whistles and speech make sine-wave utterances similar to the so-called "duplex" phenomena (Rand, 1974; Liberman, 1982), in which some portion of the stimulus (e.g. an isolated formant transition) is interpreted both as part of speech and as an additional source. For instance, Gardner and Darwin (1986) showed that the application of frequency modulation to a harmonic near to a formant in a synthetic vowel caused the harmonic to stand out perceptually although it continued simultaneously to contribute to the vowel percept.

A third example of the powerful ability of the auditory system to interpret highly stylized stimuli as speech comes from the "temporal compounds" described by Warren et al. (1990, 1996). The later study employed looped vowel sequences, each formed from a random concatenation of six $70 \mathrm{~ms}$ synthetic vowels. When the resulting token was 
played repeatedly with no inter-token silence, listeners could no longer identify the individual vowels or their order. Instead, the sequence fused into a temporal compound in which listeners often heard two simultaneous voices pronouncing syllable sequences. Rather than abandoning a speechbased interpretation, the auditory system appears to reconcile the contradictory speech cues by relaxing the constraint that they be interpreted as a single voice. Although inter-subject agreement over the syllable identities was not particularly strong, syllables were consistently drawn from the set commonly used in the native language of the listener; thus speakers of different languages could have distinctly different perceptions of the same stimulus. These results make an interesting contrast to the phonemic restoration described above: Phonemic restoration draws upon the signal context local to the deletion, in combination with linguistic constraints, to form an interpretation. In temporal compounds, however, the local cues are largely invalid (since the signal is not in fact real speech), so interpretation falls back on longer-term constraints such as the listener's native syllabary.

Studies like these reveal the auditory system's presumption that a signal with any speech-like character is indeed speech, invoking a wide range of constraints derived from language structure and the content of the message. These constraints can form a powerful basis for overcoming distortions and masking in the original signal. We now describe computational models that have addressed the application of expectations and other high-level constraints in the interpretation of auditory scenes.

\subsection{Models}

\subsubsection{Blackboards and explanation-based systems}

The perceptual phenomena described above highlight the importance of stored knowledge and expectations in permitting the interpretation of sound. A popular approach in modeling has been to use collections of knowledge sources encapsulating specific, limited aspects of the necessary knowledge, and able to act independently to solve the larger explanation problem. Knowledge sources typically cooperate through a common data structure, called a "blackboard". Several CASA systems have been built around blackboard architectures (Carver and Lesser, 1992; Nawab and Lesser, 1992; Cooke et al., 1993; Nakatani et al., 1998; Ellis, 1996; Klassner, 1996; Godsmark and Brown, 1999). Blackboards support an arbitrary combination of data-driven (bottom-up) and hypothesis-driven (top-down) activity, making them suitable for incorporating higher-level knowledge into the source separation task. For example, the highest representational level of Klassner's system is a set of "source-scripts", which embody the temporal organization of source sequences such as the regular patterning of footfalls.

One common feature of the blackboard models is the importance placed on generating consistent explanations for all the acoustic evidence. Nakatani et al. (1998) call their system a "residue-driven" architecture. Events (in their case, groups of harmonically related elements) are continuously tracked, and predictions about the immediate future are made. These predictions are compared with the actual outcome and the discrepancy, or residue, is computed by subtracting the prediction from the remaining mixture. Residues require explanation, often by the creation of new trackers. In this way, their scheme embodies the old-plus-new principle.

Klassner's (1996) blackboard system also focuses on discrepancies between observed signal features and those required for consistency with the current explanation. However, in his case the discrepancies were resolved either by modifying the explanation or by changing the parameters of the front-end signal-processing algorithms from which the features are obtained. Since optimal values for factors such as filter bandwidths and energy thresholds depend on the details of the conjunction of sources present, his system places those parameters within the control of the blackboard procedures - in contrast to the fixed single-pass signal-processing employed in other models. His system comprises a dual search in explanation space and signal-processing parameter space to find the best explanation for a given sound scene in terms of 39 abstract templates for everyday sounds such as "car engine" and "telephone ring". 
Ellis's (1996) thesis presents "prediction-driven CASA" as an alternative to the data-driven systems described in Section 5. Motivated more closely by auditory realism than the other blackboard systems, his system constructs accounts of the input sound in terms of "generic sound elements" to act as the link between raw signal properties and abstract source descriptions. Most earlier systems for CASA were limited to the separation of voiced sounds, which was reflected in their choice of representations such as tracked partials. Ellis's system sought to model unvoiced sources such as noise bursts or impulses through an expansion of its representational vocabulary. The uncertainty implicit in modeling noise signals further led to a system that could tolerate hypotheses for which direct evidence might be temporarily obscured, a framework consistent with the induction phenomena described in Section 6.1. Periodic sounds are treated as a special case, with a correlogram-based pitch tracker triggering the creation of "wefts" (Ellis, 1997) that provide estimates of the energy in each frequency channel for the modulation period, as specified in the pitch track part of the element. The number and timing of events identified by Ellis's system were in good agreement with the sources identified by listeners in ambient sound examples such as "city street" (see Fig. 6).

Motivated by the goal of reproducing complex perceptual phenomena such as ambiguity and restoration, blackboard-based systems have the

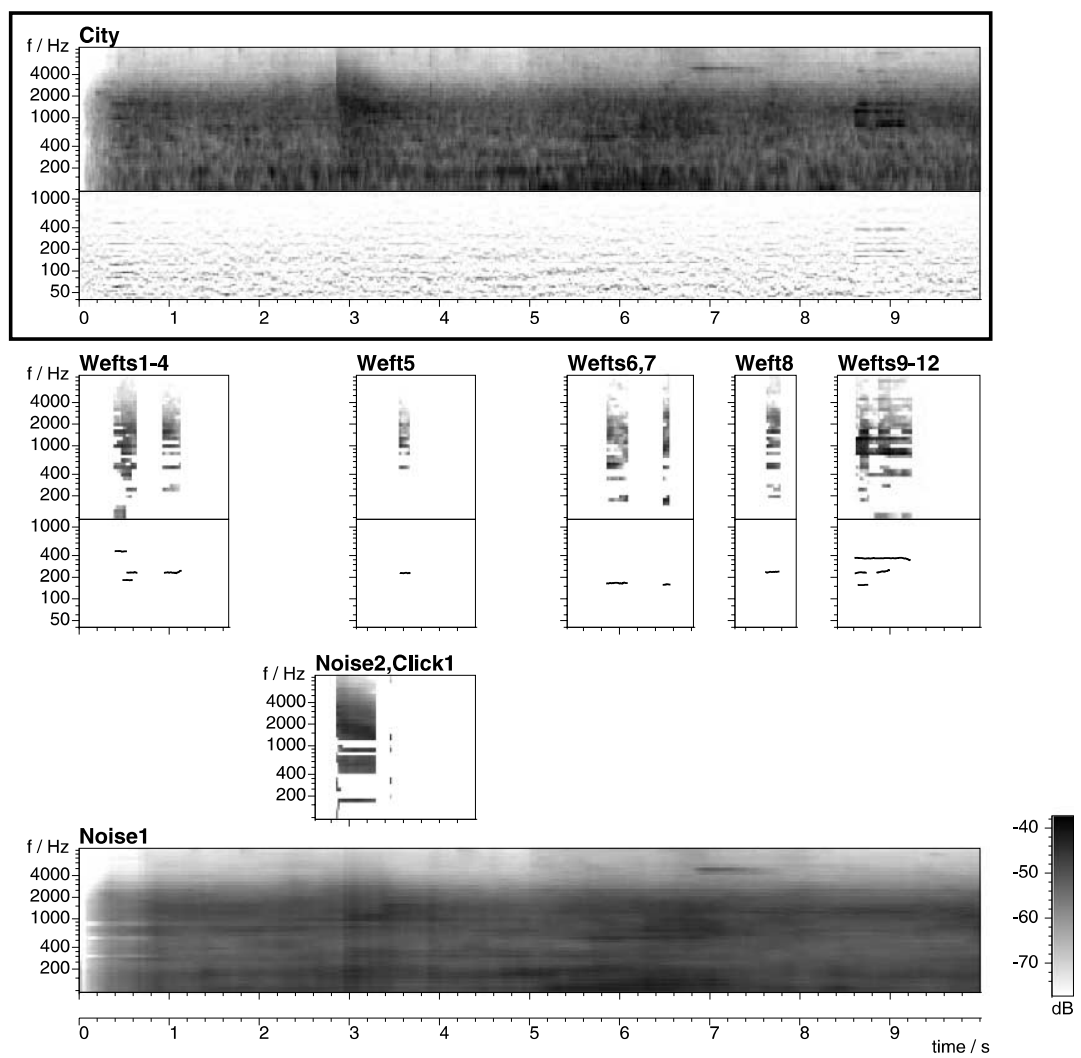

Fig. 6. Example figure adapted from Ellis (1996). The top panel shows a 10 s excerpt of "city-street ambience" represented by an auditory spectrogram as well as a periodogram (summary autocorrelation as a function of time) indicating the dominant periodicites at each point in the signal. The partial spectrograms below (labeled as Wefts, Clicks and Noise) are the generic elements postulated by the system to construct an explanation for the input mixture. Weft elements have both a partial spectrogram, showing their energy distribution, and a pitch track indicating their periodicity; Noise and Click elements are aperiodic. 
potential to produce very complex behavior from the interaction of their abstract rules. However, crafting the knowledge bases is a slow and difficult art, which offers no obvious solution to unrestricted, full-scale problems. Progress in fields such as speech recognition suggests the superiority of 'fuzzier' techniques in modeling perceptual interpretation tasks, and in particular the value of exploiting training data to tune system parameters. There are also more rigorously motivated approaches to the problem of integrating widely disparate sources of knowledge. For example, the OPTIMA system of Kashino et al. (1998) approaches the problem of analyzing complex acoustic signals - in their case, polyphonic music through the probabilistic framework of Bayesian networks.

\subsubsection{Integration with speech recognition}

Computational auditory scene analysis offers a possible solution to the serious challenges of robust automatic speech recognition. Current approaches to robust ASR (reviewed in Gong, 1995; Junqua and Haton, 1996) are far less flexible than those employed by listeners; compelling evidence of this is presented by Lippmann (1997). In addition to the variability caused by reverberation and channel distortion, recognizers in real-life environments have to cope with the non-stationarity of both target and interfering sources and uncertainty over the number of sources present. CASA is attractive because it makes few assumptions about the nature and number of sources present in the mixture, relying only on general properties of acoustic sources such as spectral continuity, common onset of components, harmonicity, and the various other potential grouping cues described in earlier sections.

Several attempts have been made to integrate CASA with ASR. The most common approach uses CASA as a sophisticated form of speech enhancement, relying on an unmodified speech recognizer to do the rest. For instance, Weintraub (1985) passed separate resynthesized signals to a hidden Markov model speech recognizer. Similarly, Bodden (1995) used binaural preprocessing prior to ASR. The main attraction of the speech enhancement route is that it allows use of existing criteria in assessing the performance of a system including CASA. As an alternative to assessment via SNR improvements and ASR recognition rates, listening tests can measure the intelligibility and naturalness of CASA-enhanced speech.

The enhancement-only application of CASA has been much criticized of late (see, for example, Bregman, 1995; Ellis, 1996; Slaney, 1998; Cooke and Green, in press) - although the weakness was certainly recognized as early as Weintraub (1985). Slaney (1998) presents a "critique of pure audition" in which he argues against a purely datadriven approach to auditory scene analysis, inspired by an analysis of top-down pathways and processes in vision (Churchland et al., 1994). Bregman (1995) too has warned against the "airtight packaging" of segregation as a preliminary to recognition, invoking duplex perception of speech as an instance where recognition overrides segregation, "defeating the original purpose of bottomup ASA" (see Fig. 7).

An alternative approach to the integration of CASA and ASR has been proposed by Cooke et al. (1994)). This scheme relies on CASA to produce an estimate of spectro-temporal regions dominated by one or other source in a mixture, and applies missing data techniques to recognize the incomplete pattern. It fits naturally with channel selection schemes such as that of Meddis and Hewitt (1992) discussed in relation to doublevowel identification. Channel selection is further inspired by neurophysiological oscillator models discussed in Section 7. The missing data strategy works on the assumption that redundancy in the speech signal allows successful recognition even when moderate amounts of the signal are corrupted or obscured. Robust recognition performance in the face of missing data can be obtained, and further improvements are possible when models of auditory spectral induction (Warren et al., 1997) are incorporated (Green et al., 1995; Morris et al., 1998). In a similar vein, Berthommier et al. (1998) incorporate CASAstyle information into speech recognition by varying the weights of separately processed frequency bands in a multi-band recognizer (Bourlard et al., 1996). 


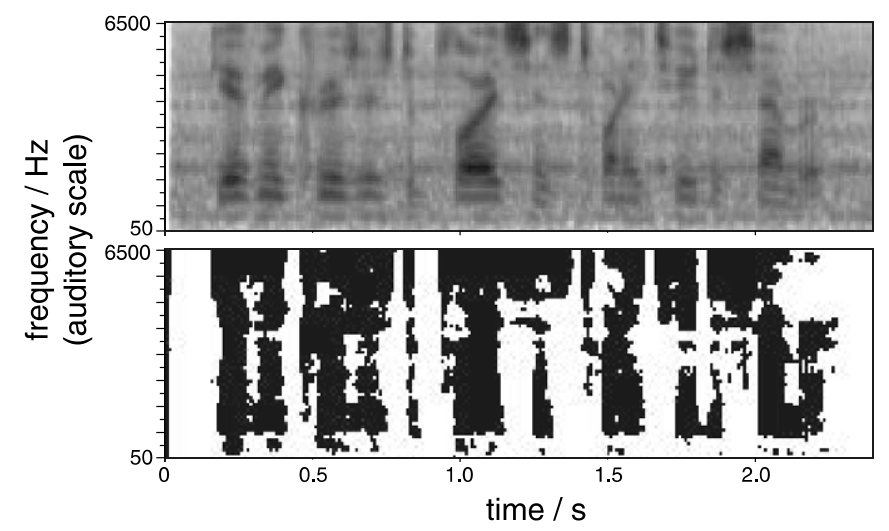

Fig. 7. The upper panel shows an auditory spectrogram for the utterance "Give me cruisers deployed since twenty two December" mixed with Lynx helicopter noise at a global SNR of $18 \mathrm{~dB}$. Dark regions of the lower panel indicate those areas where the local SNR is positive. Attempts to recognize the mixture with a conventional recognizer yielded "Is Hornes four December" while use of missing data techniques via the lower mask produced "Give cruisers deployed seventh December".

Auditory induction - or, more generally, the effect of perceived auditory continuity - has motivated a number of CASA systems. Ellis (1993) argued that restoration would be necessary to overcome obscured features in data-driven systems, and his system makes the inference of masked regions a central part of the predictionreconciliation analysis (Ellis, 1996). Okuno et al. (1997) described a scheme in which the residue remaining after extracting harmonically related regions is substituted in those temporal intervals in which no harmonic structure could be extracted, arguing that this residual is a better guess for the continuation of the voicing than silence would be - since, at the very least, it will permit induction in listeners faced with the resynthesized signal.

Ellis (1999) makes a specific proposal for incorporating speech recognition within scene analysis. Extending his prediction-driven approach, he includes a conventional speech recognition engine as one of the "component models" that can contribute to the explanation of a scene. An estimate of the speech spectrum, based on the labeling from the speech recognizer, is used to guide the analysis of the remainder of the signal by non-speech models. This re-estimation of each speech and non-speech component can be iterated to obtain stable estimates.

\subsection{Discussion}

\subsubsection{The significance of expectations}

This section has focussed on the role of expectations and abstract knowledge in auditory perception, and on efforts to model these effects. There are important implications from the demonstration that, in the absence of adequate direct cues, the auditory system will employ information from elsewhere to build its interpretation of a scene - and, as seen in the original Warren (1970) experiments, restored information is consciously indistinguishable from direct evidence. Given the enormous power of high-level constraints to restrict the range of interpretations that need be considered, the auditory system might be inclined to rely on inference in many circumstances besides those in which information is absolutely unavailable - it might be easier to 'guess' than to 'measure' when the confidence guessing is very high. Perception exists as a compromise between finding direct evidence of particular sources and the mere absence of observed contradictory evidence.

\subsubsection{Retroactivity}

Certain perceptual phenomena, starting with the phonemic restorations which depended on a later keyword (Warren and Warren, 1970), but 
including much simpler signals such as noise bands of abruptly alternating bandwidths (Bregman, 1990), show that the interpretation of a sound must sometimes wait for as much as several hundred milliseconds or longer before it can be finally decided. Examples such as the Reynolds-McAdams oboe (Mellinger, 1991) illustrate an initial organization which is consciously revised, i.e. the listener is aware of the change in organization. Blackboard systems such as those of Klassner (1996) and Ellis (1996) that maintain multiple alternative hypotheses can exhibit backward influence in certain circumstances; the system of Godsmark and Brown (1999) explicitly increases the size of its decision window until ambiguity can be resolved. Ultimately, models may need, in exceptional circumstances, to revise decisions that were previously considered complete, although it is not clear at what level of abstraction this reassessment might apply.

\section{Issues in models of auditory organization}

\subsection{Levels of explanation}

Marr's (1982) analysis of vision identified three distinct levels of explanation for any perceptual information processing task. At the lowest level is the implementation, concerned with the mechanism by which particular features are calculated or processes performed. Above this lies the level of algorithm and representation, describing a particular computational approach, capable of implementation in a variety of ways, for instance on a digital computer or in a biological realization. Marr placed particular emphasis on the highest level, which he termed the "computational theory", involving the underlying physical properties of the domain which make possible a solution to the perceptual problem. Marr used this analysis to argue that it is vital to be clear about which level any explanation is targetted, and that it is damaging to conduct research into perceptual systems without being clearly aware of the computational theory. He cited examples of research which he considered essentially wasted effort owing to the absence of a computational theory.
Numerous researchers in audition have found inspiration in Marr's work. For instance, Unoki and Akagi (1999) make a careful effort to formulate Bregman's principles mathematically to meet Marr's requirements for a computational theory. It has, however, proved difficult to find consensus over the precise nature of a computational theory to underlie auditory organization (or, for that matter, vision), and it remains an open question to find a suitable formalization of profound constraints such as the continuity and independence of acoustic sources.

\subsection{The goal of computational auditory scene analysis}

The common goal of CASA systems is the intelligent processing of sound mixtures, but individual systems differ both in the kind of sounds that are handled and in the information about them which is extracted. Some approaches seek to pluck a particular signal out of an interference whose properties are essentially ignored (e.g. the enhancement of the target voice in Brown, 1992), while others are concerned with making a complete explanation of all components in the acoustic mixture (e.g. Ellis, 1996). The former 'target enhancement' approach pursues algorithms able to handle a very wide range of conditions since it makes the fewest assumptions (e.g. only that the interference will be lower in energy than the target over a significant portion of the time-frequency plane). By contrast, 'complete explanation' accepts the added complexity of characterizing portions of the signal that are to be discarded, in the belief that this is necessary to reproduce human-style contextadaptive processing in which the interpretation of a target is influenced by non-target components. Such influences include the requirement of a plausible masker (Warren et al., 1972).

\subsection{Evaluation}

Systems that resynthesize an enhanced version of the target sound are amenable to evaluation via listening tests. Most CASA systems possess one or more internal source representations which can be used for resynthesis. It has been argued that an 
adequate model should represent all the perceptually significant information about a sound, and be able to resynthesize sources without further reference to the original mixture (Ellis, 1996). This approach should in theory be able to separate sounds even when they overlap in both time and frequency - something that resynthesis based on selective filtering (such as Brown, 1992) cannot achieve. However, the distortions associated with highly non-linear analysis and resynthesis techniques present formidable challenges in creating high-quality output. Mistakes in grouping assignments often become very prominent in resyntheses; although this can be uncomfortable for the modeler, it also carries a diagnostic benefit.

The systems of Cooke (1991) and Brown (1992) were both evaluated through a calculation of the SNR improvement on test mixtures. Since energy in an output signal cannot be directly associated with a single input component, both evaluations posed a correspondence problem. Cooke classified his "strand" elements according to their similarity to elements derived from the separate input components, whereas Brown was able to calculate the attenuation from his time-frequency mask for target and interference presented in isolation. Ellis (1996) sought a more perceptual measure of separation success by conducting listening tests in which subjects were asked to rate, on a subjective scale, the resemblance of resynthesized components to the individual sources they heard in the full original mixture.

Other approaches to evaluation include speech recognition and intelligibility scores (Weintraub, 1985; Bodden, 1995; Okuno et al., 1997), and simulations or equivalents of psycho-acoustic tests such as forced-choice discrimination.

Unlike large-vocabulary automatic speech recognition or message understanding, computational auditory scene analysis lacks a formal evaluation infrastructure at present. This makes it difficult to gauge strengths and advances both within the CASA community and between the various alternative approaches to the problem of understanding sound mixtures. One suggestion for evaluation comes from Okuno et al. (1997), who propose the simultaneous transcription of three speakers, so chosen because it guarantees that the average SNR will be below zero. This challenging problem is interesting because it will clearly reward the integration of scene analysis with speech recognition systems, although its focus on speech may bypass the issues of 'environmental sound' recognition that some see as more fundamental (Ellis, 1996).

\subsection{Neurophysiological plausibility}

A contentious question in neurophysiology is how, in neural systems, features from the same source are marked as belonging together. von der Malsburg and Schneider (1986) called this the "binding problem" and suggested a computational solution in which neurons encoding a common environmental cause are grouped by synchrony of their temporal response. This elegant proposal allows grouping to be represented "in place" without the need for separate neural structures dedicated to representing the results of grouping. Their model consists of networks of neurons whose outputs are characterized by an oscillatory pattern. They demonstrate binding of responses, marked by a common phase of oscillation, in a simple auditory example in which common onset and simultaneous activities in different frequency bands give rise to grouping between the channels. Their proposal also allows an attentional mechanism to strobe the temporal pattern and get an unobstructed, if incomplete, view of the attended source (Crick, 1984). These ideas have been actively researched in vision, where a similar binding problem exists for object segregation. These investigations have received added impetus from physiological studies which appear to show that visual stimuli can elicit synchronized oscillations across disparate regions of the visual cortex (Gray et al., 1989). Although specific evidence of visual binding through oscillations has yet to appear, the mechanism retains its attraction.

Liu et al. (1994) applied neural oscillator models to speech recognition. Strictly, their model does not involve auditory processing, but can nevertheless be interpreted as a mechanism for schemadriven grouping. The model encodes local peaks in a sharpened mel-scale LPC spectrum as independent sets of oscillations which they assume correspond to vowel formants. These oscillations 
interact with an associative memory in which formant-vowel associations are hard-wired. Reciprocal top-down and bottom-up activation leads to synchronized oscillations in those spectral regions which globally correspond to a known vowel.

Recently, a number of studies have sought to construct an account of auditory grouping phenomena in terms of neural oscillators (see Brown et al., 1996, for a review). Brown and Cooke (1998) presented an oscillator model which encompasses a number of streaming phenomena, including grouping by frequency and temporal proximity, the temporal build-up of streaming, grouping by common onset, and grouping by smooth frequency transitions. The same model, operating on a different input representation, can also account for grouping by common fundamental (Brown and Cooke, 1995), and at the same time provide an adequate explanation for the interaction of onset asynchrony and harmonicity (Ciocca and Darwin, 1993). Wang and Brown (1999) recently extended the oscillatory framework to sentence-level segregation.

Neural oscillators have been particularly successful at modeling the interaction of cue combinations, such as common onset and proximity. This is partly due to the limited vocabulary of neural architectures, in which information can only be represented as activations and weights, and thus different cues are necessarily expressed in forms that can be combined. By contrast, a traditional symbolic model of grouping might represent periodicity and onset time attributes quite separately, requiring both to be further mapped to some 'grouping strength' axis before their interaction could be considered.

\subsection{Adaptation to context and handling ambiguity}

A single fragment can serve widely differing roles depending on its surroundings and other predispositions of the interpreting system. Auditory organization models must ultimately include a stage of processing that varies according to some notion of context, but there is a wide range of practice where this stage is placed. Ambiguous signals, whose correct interpretation is not imme- diately clear, form an interesting test of contextadaptation.

Double-vowel identification models may have a simple processing sequence with no adaptation or feedback. However, once the time dimension is incorporated, the organization of the acoustic information at each instant will depend on the immediately preceding context. At the very least, the top-level groupings must reflect the accumulation of grouping cues between the different sound elements generated by the lower levels of processing, as in (Cooke, 1991, 1993; Mellinger, 1991).

Other systems have intermediate representations, which, for an identical signal, can vary in response to contextual factors. In (Weintraub, 1985), these factors are the inferred presence of one or two voiced or unvoiced speakers, which determines how many pitches will be extracted and how their associated spectra will be derived. The system of Ellis (1996) is concerned with signals that may lack any periodicity cues, in which case the division of energy into representational units can only be made according to the prevailing scene interpretation. Finally, in (Klassner's, 1996) system, the dependence of the feature extraction routines on the high-level analysis means that the representation of the same signal may vary considerably as a result of neighboring source hypotheses.

Greater degrees of context-adaptation imply more sophisticated approaches to ambiguity and to the timing of decisions about organization. The rigid signal models and powerful signal processing of Nakatani et al. (1998) permit each signal frame to be incorporated into the representation as soon as it is acquired. Other systems can delay making grouping decisions for newly detected energy to allow the accumulation of disambiguating information. In (Mellinger, 1991), the delay is a fixed latency before a new harmonic is assigned to a cluster. Brown (1992) operated in two passes, with the grouping decisions made on the intermediate elements only when they were completely formed, and all information was available. Weintraub (1985) had a different two-pass structure, with the voice extraction depending on the overall best path from the initial dynamic-programming doublevoice-state determination. 
Rather than waiting for a unique solution to appear, some systems handle ambiguity by pursuing multiple hypotheses (Ellis, 1996; Klassner, 1996; Godsmark and Brown, 1999). Although this approach is computationally expensive, it perhaps resembles listeners by maintaining a set of "current beliefs' for a partially observed signal; in real-world situations, one may not have the luxury of waiting for signal to end before commencing analysis. Listeners' interpretation of complex signals might be best understood via the incremental influence of each additional signal cue (as in the alternating noise bands of Fig. 5); ultimately, a correct understanding of human sound organization will probably include a combination of deferral, alternative hypotheses and hypothesis revision.

\subsection{Representing and employing constraints}

Since the problem of separating one signal into multiple sub-components has, in its simplest form, infinitely many solutions, the problem of auditory scene analysis may be viewed as defining and applying suitable constraints to choose a preferred alternative. The nature of these constraints, and the ways in which they are encoded and applied, form a further axis on which to distinguish between the computational models.

Each of the cues in the summary of Table 1 corresponds to a constraint, i.e. an assumption of restrictions on the form of sound emitted by realworld sources. Thus the cue of harmonicity arises because many sound sources generate matched periodic modulation across wide-frequency ranges, and the consequent constraint is that frequency bands exhibiting matched modulation patterns should be regarded as carrying energy from a single source.

In Brown's (1992) system, harmonicity and synchronized onset are expressed directly in the intermediate representation, and thus the 'knowledge' of the constraints is implicit in the computational procedure rather than being explicitly represented. By contrast, many perceptually important constraints - such as characteristic patterns of an individual's native tongue - are more arbitrary, and must be acquired and recalled, rather than simply computed. This is seen in the templates of Klassner (1996), which allow his system to have a somewhat abstracted idea of what, for instance, a telephone ring or a hair dryer sounds like. The system then uses the constraint that any scene must be explained in terms of known objects as a way to overcome the intrinsic uncertainty of a complex mixture. Unoki and Akagi (1999) formalize Bregman's 'heuristic regularities' as a series of constraints, which they deploy in their general-purpose auditory scene analysis system.

One glaring difference between computational models and real listeners is the ability of the latter to learn many of their constraints simply through exposure to the world. Future computer models may exhibit this kind of learning, but await a more detailed understanding of the nature of this process.

\subsection{Comparison with other approaches to source separation}

CASA is not the only approach to the source separation problem. Three distinct alternatives are non-auditory signal processing methods, modelbased source decomposition and blind separation.

Non-auditory signal processing methods typically make use of similar or identical cues to those employed in CASA systems, but operate without auditory inspiration or constraint. For instance, in systems of this kind (Parsons, 1976), the harmonicity cue can access frequency spectra (based perhaps on narrow-band FFTs) which have a larger number of resolved harmonics than is available with auditory frequency resolution. Denbigh and Zhao (1992) describe another narrow-band pure signal processing system which combines binaural and fundamental frequency cues.

Model-based source decomposition involves finding the optimal explanation for a number of simultaneous sources in terms of prior models for each of the sources. In HMM decomposition (Varga and Moore, 1990), a mixture of two sources is decoded by determining the most probable pairing of HMM states as a function of time. HMM decomposition requires models for all constituent sources and is computationally ex- 
pensive when both source models have a realistic number of states. The technique also requires the number of sources to be fixed in advance. Modelbased decomposition can be considered as an implementation of a totally schema-driven approach to CASA.

Blind separation (BS) techniques are motivated by the statistical independence of sources in a mixture (Comon, 1994; Bell and Sejnowski, 1995). They attempt to invert the mixing process without prior knowledge of the statistical distribution of the component signals. At present, BS is very effective under certain conditions. These include the assumption that the number of component signals is known and fixed, that their temporal alignment is known, that the mixing process is linear and constant, and that there are at least as many sensors as signals. This collection of conditions represents an ideal which is never obtained in natural listening conditions. Consequently, much of the current research effort in blind separation is aimed at relaxing some of these constraints (e.g. Torkkola, 1998; Lee et al., 1997).

van der Kouwe et al. (1999) compared the CASA and BS approaches to speech separation using the corpus of sound mixtures developed by Cooke (1991, 1993). They measured the SNR of the target speech signals before and after segregation, and found that while the chosen BS algorithm (Cardoso, 1997) typically produced a larger improvement than the representative CASA system (Wang and Brown, 1999) on broad-band noise sources, the CASA system worked best on narrowband noise sources such as tones and sirens. However, a meaningful comparison is difficult since the BS system utilized pairs of signal mixed in differing proportions (to simulate a pair of sensors), while the CASA system required just the single mixed signal. van der Kouwe et al. (1999) concluded that CASA systems operated under fewer constraints (and hence are applicable in a wider range of listening situations) than current blind separation algorithms.

\subsection{Conclusion}

In the past three decades, auditory organization has come to be recognized as an essential aspect of everyday listening. Experimental investigations have employed increasingly complex stimuli ranging from repeated tone sequences to double vowels. Further work is required to improve our understanding of sound separation of arbitrary sources in realistic environments. Nevertheless, systems which draw inspiration from the perceptual task faced by listeners have shown some success on difficult problems. Applications in domains such as robust automatic speech recognition and automated polyphonic music understanding are starting to appear. The goal of general-purpose automated sound scene understanding remains a challenging computational problem.

\section{Acknowledgements}

We wish to thank three anonymous reviewers for their insightful comments, and in particular Brian Moore for his very detailed and helpful reviews. We would also like to thank Guy Brown, Alain de Cheveigné, Stuart Cunningham, Phil Green and Steve Greenberg for useful feedback on earlier drafts.

\section{Appendix A. Resources for auditory scene analysis}

In addition to Bregman's (1990) book, useful reviews of auditory organization can be found in (Darwin and Culling, 1990; Darwin and Carlyon, 1995; Moore, 1997, Chapter 7; Handel, 1989). Volume 336 (1992) of the Philosophical Transactions of the Royal Society of London, Series B is devoted to the psychophysics of concurrent sound perception.

In 1995, the first international conference specifically concerned with computational models of auditory scene analysis processes was held in Montreal as a research workshop associated with the International Joint Conference on Artificial Intelligence. The proceedings of that meeting (Montreal, 1995) and the subsequent book (Rosenthal and Okuno, 1998) provide an illustrative cross-section of the diverse approaches to CASA which now prevail. A second CASA Workshop (Nagoya, 1997) documents further recent advances in this area. Revised papers from 
that meeting constitute a special issue of Speech Communication (1999, Vol. 3/4). A third CASA workshop was held in Stockholm in August 1999. Other computational perspectives can be found in (Cooke and Brown, 1994; Summerfield and Culling, 1995; Bregman, 1995; Slaney, 1998).

Demonstrations: A CD entitled Demonstrations of auditory scene analysis (Bregman and Ahad, 1995) contains many audio examples demonstrating the principles governing auditory scene analysis. The CD can be ordered from The MIT Press, 55 Hayward Street, Cambridge, MA 02142, USA. Interactive software demonstrations of many of the effects described in this review are part of the MATLAB Auditory Demos package which may be downloaded from http://www.dcs.shef.ac.uk/ $\sim$ martin.

Corpora: To date, computational auditory scene analysis has not required corpora of the scale typically used in automatic speech recognition. Existing speech and noise corpora have been used to create acoustic mixtures suitable for computational auditory scene analysis. For instance, the NOISEX database (Varga et al., 1992) provides a limited set of noise signals. Corpora produced by post-hoc signal combination are less than ideal, and demonstrate none of the conversational effects or compensations which occur in real spoken communication. Two corpora of conversational speech which address this limitation are available. The Map Task corpus (Thompson et al., 1993) provides recordings of several two-person conversations and contains a limited amount of overlapping speech. The ShATR (Sheffield-ATR) corpus (Karlsen et al., 1998), designed specifically for research in computational auditory scene analysis, involves five participants solving two crossword puzzles in pairs (the fifth person acts as a hint-giver). This task generates overlapped speech for nearly $40 \%$ of the corpus duration. Eight microphones provide simultaneous digital recordings from a binaurally wired mannikin, an omnidirectional pressure zone mike and five closetalking microphones, one for each participant.

More information is available on these databases at the following URLs:

NOISEX: http://svr-www.eng.cam.ac.uk/comp. speech/Section1/Data/noisex.html
Map Task: http://www.hcrc.ed.ac.uk/dialogue/ maptask.html

ShATR: http://www.dcs.shef.ac.uk/research/ groups/spandh/pr/ShATR/ShATR.html

100 mixture set used in many CASA studies: http://www.dcs.shef.ac.uk/ martin

\section{References}

Anstis, S., Saida, S., 1985. Adaptation to auditory streaming of frequency modulated tones. Journal of Experimental Psychology: Human Perception and Performance 11, 257-271.

Assmann, P.F., Summerfield, Q., 1990. Modeling the perception of concurrent vowels: vowels with different fundamental frequencies. Journal of the Acoustical Society of America 88 (2), 680-697.

Assmann, P.F., Summerfield, Q., 1994. The contribution of waveform interactions to the perception of concurrent vowels. Journal of the Acoustical Society of America 95 (1), 471-484.

Assmann, P.F., Summerfield, Q., in press. The perception of speech under adverse acoustic conditions. In: Greenberg, S., Ainsworth, W. (Eds.), The Auditory Basis of Speech Perception. Springer, Berlin.

Bailey, P.J., Summerfield, A., Dorman, M., 1977. On the identification of sine-wave analogues of certain speech sounds. Report no: SR-51/52, Haskins Labs.

Beauvois, M.W., Meddis, R., 1991. A computer model of auditory stream segregation. Quarterly Journal of Experimental Psychology 43A (3), 517-541.

Beauvois, M.W., Meddis, R., 1996. Computer simulation of auditory stream segregation in alternating tone sequences. Journal of the Acoustical Society of America 99 (4), 2270 2280 (Pt. 1).

Bell, A.J., Sejnowski, T.J., 1995. An information maximisation approach to blind separation and blind deconvolution. Neural Computation 7 (6), 1129-1159.

Berthommier, Meyer, 1997. A model of double-vowel segregation with AM map and without F0 tracking. In: Proceedings of the Second Workshop on Computational Auditory Scene Analysis, International Joint Conference on Artificial Intelligence, Nagoya.

Berthommier, F., Glotin, H., Tessier, E., Bourlard, H., 1998. Interfacing of CASA and partial recognition based on a multistream technique. In: Proceedings of the International Conference on Spoken Language Processing, Sydney.

Bird, J., Darwin, C.J., 1998. Effects of a difference in fundamental frequency in separating two sentences. In: Palmer, A.R., Rees, A., Summerfield, A.Q., Meddis, R. (Eds.), Psychophysical and Physiological Advances in Hearing. Whurr, London, pp. 263-269.

Bodden, M., 1995. Binaural modeling and auditory scene analysis. In: Proceedings of the IEEE Workshop on Applications of Signal Processing to Audio and Acoustics, Mohonk. 
Bourlard, H., Dupont, S., Hermansky, H., Morgan, N., 1996. Towards sub-band-based speech recognition. In: Proceedings of the European Signal Processing Conference, Trieste, pp. $1579-1582$.

Bregman, A.S., 1978. Auditory streaming is cumulative. Journal of Experimental Psychology: Human Perception and Performance 4, 380-387.

Bregman, A.S., 1984. Auditory scene analysis. In: Proceedings of the Seventh International Conference on Pattern Recognition, Silver Spring, MD.

Bregman, A.S., 1990. Auditory Scene Analysis: The Perceptual Organization of Sound. MIT Press, Cambridge, MA.

Bregman, A.S., 1995. Use of psychological data in building ASA models. In: Proceedings of the IEEE Workshop on Applications of Signal Processing to Audio and Acoustics, Mohonk.

Bregman, A.S., Ahad, P., 1995. Demonstrations of Auditory Scene Analysis: The Perceptual Organisation of Sound. MIT Press.

Bregman, A.S., Campbell, J., 1971. Primary auditory stream segregation and perception of order in rapid sequences of tones. Journal of Experimental Psychology 89, 244-249.

Bregman, A.S., Pinker, S., 1978. Auditory streaming and the building of timbre. Canadian Journal of Psychology 32, 1931.

Bregman, A.S., Rudnicky, A., 1975. Auditory segregation: stream or streams? Journal of Experimental Psychology: Human Perception and Performance 1, 263-267.

Bregman, A.S., Abramson, J., Doehring, P., Darwin, C.J., 1985. Spectral integration based on common amplitude modulation. Perception and Psychophysics 37, 483-493.

Bregman, A.S., Liao, C., Levitan, R., 1990. Auditory grouping based on fundemental frequency and format peak frequency. Canadian Journal of Psychology 44 (3), 400-413.

Broadbent, D.E., Ladefoged, P., 1957. On the fusion of sounds reaching different sense organs. Journal of the Acoustical Society of America 29, 708-710.

Brokx, J.P.L., Nooteboom, S.G., 1982. Intonation and the perceptual separation of simultaneous voices. Journal of Phonetics 10, 23-36.

Brown, G.J., 1992. Computational auditory scene analysis: a representational approach. Unpublished doctoral thesis (CS-92-22), Department of Computer Science, University of Sheffield.

Brown, G.J., Cooke, M.P., 1994. Computational auditory scene analysis. Computer Speech and Language 8, 297-336.

Brown, G.J., Cooke, M.P., 1995. A neural oscillator model of primitive audio grouping. In: Proceedings of the IEEE Workshop on Applications of Signal Processing to Audio and Acoustics, Mohonk.

Brown, G.J., Cooke, M.P., 1998. Temporal synchronization in a neural oscillator model of primitive auditory stream segregation. In: Rosenthal, D., Okuno, H. (Eds.), Readings in Computational Auditory Scene Analysis. Lawrence Erlbaum, London.

Brown, G.J., Cooke, M.P., Mousset, E., 1996. Are neural oscillations the substrate of auditory grouping? ESCA
Tutorial and Workshop on the Auditory Basis of Speech Perception, Keele University, 15-19 July.

Buus, S., 1985. Release from masking caused by envelope fluctuations. Journal of the Acoustical Society of America 78 (6), 1958-1965.

Cardoso, J.F., 1997. Estimating equations for source separation. In: Proceedings of the ICASSP '97.

Carlyon, R.P., 1994. Further evidence against an acrossfrequency mechanism specific to the detection of frequency modulation (FM) incoherence between resolved frequency components. Journal of the Acoustical Society of America 95, 949-961.

Carver, N., Lesser, V., 1992. Blackboard systems for knowledge-based signal understanding. In: Oppenheim, A.V., Nawab, S.H. (Eds.), Symbolic and Knowledge-based Signal Processing. Prentice-Hall, Englewood Cliffs, NJ.

Cherry, E.C., 1953. Some experiments on the recognition of speech with one and with two ears. Journal of the Acoustical Society of America 25, 975-979.

Churchland, P., Ramachandran, V.S., Sejnowski, T., 1994. A critique of pure vision. In: Koch, C., Davis, J. (Eds.), Large Scale Neuronal Theories of the Brain. MIT Press, Cambridge, MA.

Ciocca, V., Darwin, C.J., 1993. Effects of onset asynchrony on pitch perception: adaptation or grouping. Journal of the Acoustical Society of America 93 (5), 2870-2878.

Cole, R.A., Scott, B., 1973. Perception of temporal order in speech: the role of vowel transitions. Canadian Journal of Psychology 27, 441-449.

Comon, P., 1994. Independent component analysis: A new concept. Signal Processing 36 (3), 287-314.

Cooke, M.P., 1991. Modelling auditory processing and organisation. Doctoral thesis, Cambridge University Press, Cambridge, 1993.

Cooke, M.P., Brown, G.J., 1994. Separating simultaneous sound sources: issues, challenges and models. In: Keller, E. (Ed.), Fundamentals of Speech Synthesis and Speech Recognition. Wiley, New York, pp. 295-312.

Cooke, M.P., Green, P.D., in press. Auditory organisation and speech perception: pointers for robust ASR. In: Greenberg, S., Ainsworth, W. (Eds.), Listening to Speech: An auditory Perspective. Oxford University Press, Oxford

Cooke, M.P., Brown, G.J., Crawford, M.D, Green, P.D., 1993. Computational auditory scene analysis: listening to several things at once. Endeavour 17, 186-190.

Cooke, M.P., Green, P.D., Crawford, M.D., 1994. Handling missing data in speech recognition. In: Proceedings of the International Conference on Speech and Language Processing, Yokohama, pp. 1555-1558.

Crick, F., 1984. Function of the thalamic reticular complex: the searchlight hypothesis. In: Proceedings of the National Academy of Sciences, Vol. 81, pp. 4586-4590.

Culling, J.F., Darwin, C.J., 1993. Perceptual separation of simultaneous vowels: within and across-for mant grouping by F0. Journal of the Acoustical Society of America 93 (6), 3454-3467. 
Culling, J.F., Darwin, C.J., 1994. Perceptual and computational separation of simultaneous vowels: cues arising from lowfrequency beating. Journal of the Acoustical Society of America 95 (3), 1559-1569.

Culling, J.F., Summerfield, Q., 1995a. The role of frequency modulation in the perceptual segregation of concurrent vowels. Journal of the Acoustical Society of America 98 (2), 837-846.

Culling, J.F., Summerfield, Q., 1995b. Perceptual segregation of concurrent speech sounds: absence of across-frequency grouping by common interaural delay. Journal of the Acoustical Society of America 98 (2), 785-797.

Culling, J.F., Summerfield, Q., Marshall, D.H., 1994. Effects of simulated reverberation on the use of binaural cues and fundamental-frequency differences for separating concurrent vowels. Speech Communication 14, 71-95.

Cutting, J.E., 1976. Auditory and linguistic processes in speech perception: inferences from six fusions in dichotic listening. Psychological Review 83, 114-140.

Darwin, C.J., 1981. Perceptual grouping of speech components different in fundamental frequency and onset-time. Quarterly Journal of Experimental Psychology 3A, 185207.

Darwin, C.J., 1984. Perceiving vowels in the presence of another sound: constraints on formant perception. Journal of the Acoustical Society of America 76 (6), 1636-1647.

Darwin, C.J., Bethell-Fox, C.E., 1977. Pitch continuity and speech source attribution. Journal of Experimental Psychology: Human Perception and Performance 3, 665-672.

Darwin, C.J., Carlyon, R.P., 1995. Auditory grouping. In: Moore, B.C.J. (Ed.), The Handbook of Perception and Cognition, Vol. 6, Hearing. Academic Press, New York, pp. 387-424.

Darwin, C.J., Ciocca, V., 1992. Grouping in pitch perception: effects of onset asynchrony and ear of presentation of a mistuned component. Journal of the Acoustical Society of America 91, 3381-3390.

Darwin, C.J., Culling, J.F., 1990. Speech perception seen through the ear. Speech Communication 9 (5-6), 469-475.

Darwin, C.J., Gardner, R.B., 1986. Mistuning a harmonic of a vowel: grouping and phase effects on vowel quality. Journal of the Acoustical Society of America 79 (3), 838-845.

Darwin, C.J., Pattison, H., Gardner, R.B., 1989. Vowel quality changes produced by surrounding tone sequences. Perception and Psychophysics 45, 333-342.

Darwin, C.J., Hukin, R.W., Al-Khatib, B.Y., 1995. Grouping in pitch perception: evidence for sequential constraints. Journal of the Acoustical Society of America 98 (2), 880 885 .

de Cheveigné, A., 1993. Separation of concurrent harmonic sounds: fundamental frequency estimation and a timedomain cancellation model of auditory processing. Journal of the Acoustical Society of America 93, 3271-3290.

de Cheveigné, A., 1997. Concurrent vowel identification III: a neural model of harmonic interference cancellation. Journal of the Acoustical Society of America 101, 2857-2865. de Cheveigne, A., 1999. Waveform interactions and the segregation of concurrent vowels. J. Acoust. Soc. Am. 106 (5), 2959-2972.

de Cheveigné, A., McAdams, S., Laroche, J., Rosenberg, M., 1995. Identification of concurrent harmonic and inharmonic vowels: a test of the theory of harmonic cancellation and enhancement. Journal of the Acoustical Society of America 97 (6), 3736-3748.

de Cheveigné, A., Kawahara, H., Tsuzaki, M., Aikawa, K., 1997a. Concurrent vowel identification I: effects of relative level and F0 difference. J. Acoust. Soc. Am. 101, 2839-2847.

de Cheveigné, A., McAdams, S., Marin, C., 1997b. Concurrent vowel identification II: effects of phase, harmonicity and task. J. Acoust. Soc. Am. 101, 2848-2856.

Deutsch, D., 1975. Two-channel listening to musical scales. Journal of the Acoustical Society of America 57, 1156-1160.

Denbigh, P.N., Zhao, J., 1992. Pitch extraction and the separation of overlapping speech. Speech Communication $11,119-125$.

Dowling, W.J., 1973. Rhythmic groups and subjective chunks in memory for melodies. Perception and Psychophysics 14, 37-40.

Duifhuis, H., Willems, L.F., Sluyter, R.J., 1982. Measurement of pitch in speech: an implementation of Goldstein's theory of pitch perception. Journal of the Acoustical Society of America 71, 1568-1580.

Durlach, N.I., 1963. Equalization and cancellation theory of binaural masking-level differences. Journal of the Acoustical Society of America 35, 1206-1218.

Ellis, D.P.W., 1993. Hierarchic models of hearing for sound separation and reconstruction. In: Proceedings of the IEEE Workshop on Applications of Signal Processing to Audio and Acoustics, Mohonk.

Ellis, D.P.W., 1996. Prediction-driven computational auditory scene analysis. Unpublished doctoral dissertation, Department of Electrical Engineering and Computer Science, Massachusetts Institute of Technology.

Ellis, D.P.W., 1997. The weft: a representation for periodic sounds. In: Proceedings of the International Conference on Acoustics. Speech and Signal Processing, Munich, pp. 13071310 .

Ellis, D.P.W., 1999. Using knowledge to organize sound: the prediction-driven approach to computatational auditory scene analysis, and its application to speech/nonspeech mixtures. Speech Communication 27 (3-4), 281-298.

Gardner, R.B., Darwin, C.J., 1986. Grouping of vowel harmonics by frequency modulation: absence of effects on phonemic categorization. Perception and Psychophysics 40 (3), 183-187.

Gardner, R.B., Gaskill, S.A., Darwin, C.J., 1989. Perceptual grouping of formants with static and dynamic differences in fundamental frequency. Journal of the Acoustical Society of America 85 (3), 1329-1337.

Godsmark, D., Brown, G.J., 1999. A blackboard architecture for computational auditory scene analysis. Speech Communication 27 (3-4), 351-366. 
Gong, Y., 1995. Speech recognition in noisy environments: a survey. Speech Communication 16, 261-291.

Gray, C.M., König, P., Engel, A.K., Singer, W., 1989. Oscillatory responses in cat visual cortex exhibit intercolumnar synchronization which reflects global stimulus properties. Nature 338, 334-337.

Green, P.D., Cooke, M.P., Crawford, M.D., 1995. Auditory scene analysis and hidden Markov model recognition of speech in noise. In: Proceedings of the International Conference on Acoustics, Speech and Signal Processing, 1995, pp. 401-404.

Guttman, N., Julesz, B., 1963. Lower limits of auditory periodicity analysis. Journal of the Acoustical Society of America 35, 610.

Hall, J.W., Haggard, M.P., Fernandes, M.A., 1984. Detection in noise by spectro-temporal pattern analysis. Journal of the Acoustical Society of America 76, 50-56.

Handel, S., 1989. Listening: An Introduction to the Perception of Auditory Events. MIT Press, Cambridge, MA.

Hartman, W.M., Johnson, D., 1991. Stream segregation and peripheral channeling. Music Perception 9 (2), 155-184.

Hill, N.J., Darwin, C.J., 1993. Effects of onset asynchrony and of mistuning on the lateralization of a pure tone embedded in a harmonic complex. Journal of the Acoustical Society of America 93, 2307-2308.

Houtgast, T., 1972. Psychophysical evidence for lateral inhibition in hearing. Journal of the Acoustical Society of America 51 (6), 1885-1894.

Howard-Jones, P.A., Rosen, S., 1993. Uncomodulated glimpsing in "checkerboard" noise. Journal of the Acoustical Society of America 93 (5), 2915-2922.

Hukin, R.W., Darwin, C.J., 1995. Effects of contralateral presentation and of interaural time differences in segregating a harmonic from a vowel. Journal of the Acoustical Society of America 98 (3), 1380-1387.

Jeffress, L.A., 1948. A place theory of sound localization. Journal of Comparative and Physiological Psychology 41, 35-39.

Jones, M.R., 1976. Time, our lost dimension: toward a new theory of perception, attention and memory. Psychological Review 83, 323-355.

Junqua, J.-C., Haton, J.P., 1996. Robustness in Automatic Speech Recognition. Kluwer Academic Publishers, Dordrecht.

Kaernbach, C., 1992. On the consistency of tapping to repeated noise. Journal of the Acoustical Society of America 92, 788793.

Karlsen, B.L., Brown, G.J., Cooke, M.P., Crawford, M.D., Green, P.D., Renals, S.J., 1998. Analysis of a simultaneousspeaker sound corpus. In: Rosenthal, D., Okuno, H. (Eds.), Readings in Computational Auditory Scene Analysis. Lawrence Erlbaum, London.

Kashino, K., Nakadai, K., Kinoshita, T., Tanaka, H., 1998. Application of the Bayesian probability network to music scene analysis. In: Rosenthal, D., Okuno, H. (Eds.), Readings in Computational Auditory Scene Analysis. Lawrence Erlbaum, London.
Klassner, F., 1996. Data reprocessing in signal understanding systems. Unpublished Ph.D. Dissertation, Department of Computer Science, University of Massachusetts, Amherst.

Lea, A., 1992. Auditory modeling of vowel perception. Unpublished doctoral thesis, University of Nottingham.

Lee, T.-W., Bell, A.J., Orglmeister, R., 1997. Blind source separation of real world signals. In: Proceedings of the IEEE International Conference on Neural Networks, 1997, pp. 2129-2135.

Liberman, A.M., 1982. On the finding that speech is special. American Psychologist 37 (2), 148-167 (reprint in: Handbook of Cognitive Neuroscience. Plenum Press, New York, 1984, pp. 169-197).

Licklider, J.C.R., 1951. A duplex theory of pitch perception. In: Schubert, D. (Eds.), Experientia 7, Dowden, Hutchinson and Ross, pp. 128-133 (reprinted in: Physiological Acoustics, 1979).

Lippmann, R.P., 1997. Speech recognition by machines and humans. Speech Communication 22 (1), 1-16.

Liu, F., Yamaguchi, Y., Shimizu, H., 1994. Flexible vowel recognition by the generation of dynamic coherence in oscillator neural networks: speaker-independent vowel recognition. Biological Cybernetics 71, 105-114.

Lyon, R.F., 1983. A computational model of binaural localization and separation. In: Proceedings of the International Conference on Acoustics, Speech and Signal Processing, Boston, pp. 1148-1151.

Marr, D., 1982. Vision. Freeman, New York.

McAdams, S., 1984. Spectral fusion, spectral parsing and the formation of auditory images. Unpublished doctoral dissertation, Stanford University.

McCabe, S.L., Denham, M.J., 1997. A model of auditory streaming. Journal of the Acoustical Society of America 101 (3), 1611-1621.

McKeown, J.D., Patterson, R.D., 1995. The time course of auditory segregation: concurrent vowels that vary in duration. Journal of the Acoustical Society of America 98 (4), 1866-1877.

Meddis, R., Hewitt, M.J., 1991. Virtual pitch and phase sensitivity of a computer model of the auditory periphery. I: Pitch identification. Journal of the Acoustical Society of America 89 (6), 2866-2882.

Meddis, R., Hewitt, M.J., 1992. Modelling the identification of concurrent vowels with different fundamental frequencies. Journal of the Acoustical Society of America 91 (1), 233245.

Mellinger, D.K., 1991. Event formation and separation in musical sound. Unpublished doctoral dissertation, Department of Music, Stanford University.

Miller, G.A., Heise, G.A., 1950. The trill threshold. Journal of the Acoustical Society of America 22, 637-638.

Montreal, 1995. In: Proceedings of the First Workshop on Computational Auditory Scene Analysis, International Joint Conference on Artificial Intelligence, Montreal.

Moore, B.C.J., 1997. An Introduction to the Psychology of Hearing, fourth ed.. Academic Press, New York. 
Moore, B.C.J., Glasberg, B.R., Peters, R.W., 1985. Relative dominance of individual partials in determining the pitch of complex tones. Journal of the Acoustical Society of America 77, 1853-1860.

Moore, B.C.J., Glasberg, B.R., Peters, R.W., 1986. Thresholds for hearing mistuned partials as separate tones in harmonic complexes. Journal of the Acoustical Society of America 80, 479-483.

Moore, D.R., 1987. Physiology of the higher auditory system. British Medical Bulletin 43 (4), 856-870.

Morris, A.C., Cooke, M.P., Green, P.D., 1998. Some solutions to the missing feature problem in data classification, with application to noise robust ASR. In: Proceedings of the International Conference on Acoustics, Speech and Signal Processing, Seattle.

Nagoya, 1997. In: Proceedings of the Second Workshop on Computational Auditory Scene Analysis, International Joint Conference on Artificial Intelligence, Nagoya.

Nakatani, T., Kashino, K., Okuno, H.G., 1997. Integration of speech stream and music stream segregation based on ontology. In: Proceedings of the Second Workshop on Computational Auditory Scene Analysis, International Joint Conference on Artificial Intelligence, Nagoya.

Nakatani, T., Okuno, H.G., Goto, M., Ito, T., 1998. Multiagent based binaural sound stream segregation. In: Rosenthal, D., Okuno, H. (Eds.), Readings in Computational Auditory Scene Analysis. Lawrence Erlbaum, London.

Nawab, S.H., Lesser, V., 1992. Integrated processing and understanding of signals. In: Oppenheim, A.V., Nawab, S.H. (Eds.), Symbolic and Knowledge-based Signal Processing. Prentice-Hall, Englewood Cliffs, NJ.

Okuno, H.G., Nakatani, T., Kawabata, T., 1997. Challenge problem: understanding three simultaneous speakers. In: Proceedings of the Second Workshop on Computational Auditory Scene Analysis. International Joint Conference on Artificial Intelligence, Nagoya.

Palmer, A.R., 1990. The representation of the spectra and fundamental frequencies of steady-state single and doublevowel sounds in the temporal discharge patterns of guinea pig cochlear-nerve fibres. Journal of the Acoustical Society of America 88 (3), 1412-1426.

Parsons, T.W., 1976. Separation of speech from interfering speech by means of harmonic selection. Journal of the Acoustical Society of America 60 (4), 911-918.

Patterson, R.D., 1987. A pulse ribbon model of monaural phase perception. Journal of the Acoustical Society of America 82 (5), 1560-1586.

Pierce, J.R., 1983. The Science of Musical Sound. Freeman, New York.

Rand, T.C., 1974. Dichotic release from masking for speech. Journal of the Acoustical Society of America 55, 678-680.

Remez, R.E., Rubin, P.E., Pisoni, D.B., Carrell, T.D., 1981. Speech perception without traditional speech cues. Science 212, 947-950.

Remez, R.E., Rubin, P.E., Berns, S.M., Pardo, J.S., Lang, J.M., 1994. On the perceptual organization of speech. Psychological Review 101 (1), 129-156.
Richards, V., 1987. Monaural envelope correlation perception. Journal of the Acoustical Society of America 82 (5), 16211630.

Rogers, W.L., Bregman, A.S., 1993. An experimental evaluation of three theories of auditory stream segregation. Perception and Psychophysics 53 (2), 179-189.

Rosenthal, D., Okuno, H., 1998. Readings in Computational Auditory Scene Analysis. Lawrence Erlbaum, London.

Saberi, K., Hafter, E.R., 1995. A common neural code for frequency and amplitude modulated sounds. Nature 374 (6522), 537-539.

Scheffers, M.T.M., 1983. Sifting vowels: auditory pitch analysis and sound segregation, Unpublished doctoral thesis, University of Groningen.

Schooneveldt, G.P., Moore, B.C.J., 1989. Comodulation masking release (CMR) as a function of masker bandwidth, modulator bandwidth, and signal duration. Journal of the Acoustical Society of America 85 (1), 273-281.

Shackleton, T.M., Meddis, R., 1992. The role of interaural time difference and fundamental frequency difference in the identification of concurrent vowel pairs. Journal of the Acoustical Society of America 91, 3579-3581.

Slaney, M., 1998. A critique of pure audition. In: Rosenthal, D., Okuno, H. (Eds.), Readings in Computational Auditory Scene Analysis. Lawrence Erlbaum, London.

Stubbs, Summerfield, 1988. Evaluation of 2 voice-separation algorithms using normal-hearing and hearing-impaired listeners. Journal of the Acoustical Society of America 84 (4), 1236-1249.

Summerfield, Q., Culling, J.F., 1992. Auditory segregation of competing voices: absence of effects of FM or AM coherence. Philosophical Transactions of the Royal Society London B 336, 415-422.

Summerfield, Q., Culling, J.F., 1995. Auditory computations which separate speech from competing sounds: a comparison of binaural and monaural processes. In: Keller, E. (Ed.), Fundamentals of Speech Synthesis and Speech Recognition. Wiley, New York.

Thompson, H., Bard, E., Anderson, A., Doherty-Sneddon, G., 1993. The HCRC map task corpus: a natural spoken dialogue corpus. In: Proceedings of the International Symposium on Spoken Dialogue, Tokyo, pp. 33-36.

Thurlow, W.R., Elfner, L.F., 1959. Continuity effects with alternately sounding tones. JASA 31, 1337-1339.

Todd, N.P.M., 1996. An auditory cortical theory of primitive auditory grouping. Network: Computation in Neural Systems 7, 349-356.

Torkkola, K., 1998. Blind signal separation in communications: making use of known signal distributions. In: Proceedings of the IEEE DSP Workshop, Bryce Canyon, UT, August $10-12$.

Unoki, M., Akagi, M., 1999. A method of signal extraction from noisy signal based on auditory scene analysis. Speech Communication 27 (3-4), 261-279.

Van der Kouwe, A.J.W., Wang, D.L., Brown, G.J., 1999. A comparison of auditory and blind separation techniques for speech segregation. Technical Report OSU-CISRC-6/99- 
TR15, Ohio State University, Department of Computer and Information Science.

van Noorden, L.P.A.S., 1975. Temporal coherence in the perception of tone sequences. Ph.D. Dissertation, Eindhoven University of Technology.

Varga, A.P., Moore, R.K., 1990. Hidden markov model decomposition of speech and noise. In: Proceedings of the International Conference on Acoustics, Speech and Signal Processing, pp. 845-848.

Varga, A.P., Steeneken, H.J.M., Tomlinson, M., Jones, D., 1992. The NOISEX-92 study on the effect of additive noise on automatic speech recognition. Technical Report, Speech Research Unit, Defence Research Agency, Malvern, UK.

Vliegen, J., Oxenham, A.J., 1999. Sequential stream segregation in the absence of spectral cues. Journal of the Acoustical Society of America 105 (1), 339-346.

Vliegen, J., Moore, B.C.J., Oxenham, A.J., 1999. The role of spectral and periodicity cues in auditory stream segregation measured using a temporal discrimination task. Journal of the Acoustical Society of America 106 (2), 938-945.

von der Malsburg, C., Schneider, W., 1986. A neural cocktailparty processor. Biological Cybernetics $54,29-40$.

Wang, D.L., Brown, G.J., 1999. Separation of speech from interfering sounds based on oscillatory correlation. IEEE Transactions on Neural Networks 10 (3), 684-697.

Warren, R.M., 1970. Perceptual restoration of missing speech sounds. Science 167, 392-393.

Warren, R.M., Bashford, J.A., 1976. Auditory contralateral induction: an early stage in binaural processing. Perception and Psychophysics 20 (5), 380-386.
Warren, R.M., Warren, R.P., 1970. Auditory illusions and confusions. Scientific American 223 (12), 30-36.

Warren, R.M., Obusek, C.J., Ackroff, J.M., 1972. Auditory induction: perceptual synthesis of absent sounds. Science 176, 1149-1151.

Warren, R.M., Bashford, J.A., Gardner, D.A., 1990. Tweaking the lexicon: organization of vowel sequences into words. Perception and Psychophysics 47 (5), 423-432.

Warren, R.M., Healy, E.W, Chalikia, M.H., 1996. The vowelsequence illusion: intrasubject stability and intersubject agreement of syllabic forms. Journal of the Acoustical Society of America 100 (4), 2452-2461.

Warren, R.M., Hainsworth, K.R., Brubaker, B.S., Bashford, J.A., Healy, E.W., 1997. Spectral restoration of speech: intelligibility is increased by inserting noise in spectral gaps. Perception and psychophysics 59 (2), 275-283.

Watkins, A.J., 1991. Central, auditory mechanisms of perceptual compensation for spectral-envelope distortion. Journal of the Acoustical Society of America 90 (6), 2942-2955.

Weintraub, M., 1985. A theory and computational model of auditory monaural sound separation. Unpublished doctoral dissertation, Department of Electrical Engineering, Stanford University.

Woods, W.S., Colburn, H.S., 1992. Test of a model of auditory object formation using intensity and interaural time difference discrimination. Journal of the Acoustical Society of America 91 (5), 2894-2902.

Zakarauskas, P., Cynader, M.S., 1993. A computational theory of spectral cue localization. Journal of the Acoustical Society of America 94 (3), 1323-1331. 